\title{
Energymomentum Complex in General Relativity and Gauge Theory
}

\author{
David Hestenes ${ }^{1}$ \\ Department of Physics and Astronomy \\ Arizona State University, Tempe, Arizona 85287-1504
}

\begin{abstract}
Alternative versions of the energymomentum complex in general relativity are given compact new formulations with spacetime algebra. A new unitary form for Einstein's equation greatly simplifies the derivation and analysis of gravitational superpotentials. Interpretation of Einstein's equations as a gauge field theory on flat spacetime is shown to resolve ambiguities in energymomentum conservation laws and reveal intriguing new relations between superpotential, gauge connection and spin angular momentum with rich new possibilities for physical interpretation.
\end{abstract}

\section{Introduction}

General relativity (GR) has recently been reformulated as a gauge theory in terms of spacetime algebra STA $[1,2,3]$. This brings new mathematical tools to the formulation and analysis of physical problems in GR as well as new ideas for refining and extending the theory. Those tools and ideas are applied in this article to long-standing problems about energymomentum localization in GR.

Though many different mathematical formalisms for GR have been proposed, the "covariant tensor formalism" originally introduced by Einstein is still the clear favorite in the literature. However, since the seminal paper on gauge theory by Weyl [6], it has been clear that orthonormal frames called tetrads, or vierbeins, are essential for incorporating the Dirac equation into GR. This involves an elaborate "tetrad formalism" that considerably complicates the classical tensor formalism, so it has remained on the fringes of mainstream GR. The "STA gauge theory formalism" employed in this paper can be regarded as a marriage of tensors and tetrads that enhances both with new algebraic methods, though it is recognized as a device to make equations coordinate-free [8]. While the classical features of GR are incorporated without change into the formalism, advantages of the new algebraic methods are illustrated in an analysis of energymomentum tensors. Thus, we have one more way to do GR. However, gauge theory provides added value to GR with a new way to incorporate a flat space background and thus interpret GR as a gravitational field theory on Minkowski spacetime without altering its mathematical content. Indeed, it unmasks the tetrad formalism as an indirect way to smuggle flat space into GR. The main purpose of this paper is to show how STA gauge theory provides new insight into the problem of defining a suitable energymomentum tensor for gravity.

\footnotetext{
${ }^{1}$ electronic mail:hestenes@asu.edu
} 
To set the stage, let us review some highlights in the troubled history of energy-momentum conservation in GR. The core of GR is Einstein's famous gravitational field equation

$$
G_{\mu \nu} \equiv R_{\mu \nu}-\frac{1}{2} g_{\mu \nu} R=\kappa T_{\mu \nu},
$$

where $\kappa=8 \pi$ in natural units, and the meaning of the terms is presumed to be known by the reader. Shortly after establishing this equation in his seminal paper of 1915, Einstein set forth a general argument for decomposing it as follows [7]:

$$
g G_{\alpha}^{\mu}=\kappa\left({ }_{E} \mathcal{T}_{\alpha}^{\mu}-g_{E} t_{\alpha}^{\mu}\right)=\kappa g T_{\alpha}^{\mu},
$$

where $g \equiv\left|\operatorname{det}\left(g_{\mu \nu}\right)\right|^{\frac{1}{2}}$. Let's call this the Einstein split of Einstein's tensor density $g G_{\mu \nu}$. Einstein interpreted $g_{E} t_{\alpha}^{\mu}$ as the gravitational energymomentum density and defined it so that

$$
\partial_{\mu E} \mathcal{T}_{\alpha}^{\mu}=0,
$$

where $\partial_{\mu}=\partial / \partial x^{\mu}$. It follows that

$$
\partial_{\mu}\left[g\left(T_{\alpha}^{\mu}+{ }_{E} t_{\alpha}^{\mu}\right)\right]=0,
$$

which Einstein interpreted as a general energymomentum conservation law.

Problems with Einstein's split began to surface immediately [10], beginning with the fact that it is not covariant because ${ }_{E} t_{\alpha}^{\mu}$ is a pseudotensor rather a tensor. Thus, ${ }_{E} t_{\alpha}^{\mu}$ has the perplexing property of vanishing in the absence of gravitation (flat space) for retangular coordinates but not for polar coordinates. And in curved space, it is always possible to select coordinates so that ${ }_{E} t_{\alpha}^{\mu}=0$ at any given point.

Furthermore, over subsequent years, many authors also noted that the precise functional form of Einstein's ${ }_{E} t_{\alpha}^{\mu}$ is not determined by a split satisfying (3), which is all one needs to get the "conservation law" (4). Thus, for any pseudotensor of the form $X_{\alpha}^{\mu \nu}=-X_{\alpha}^{\nu \mu}$, we have the identity $\partial_{\mu} \partial_{\nu} X_{\alpha}^{\mu \nu}=0$. Hence, we can define a new split of $G_{\alpha}^{\mu}$ into primed quantities $\mathcal{T}_{\alpha}^{\prime \mu}={ }_{E} \mathcal{T}_{\alpha}^{\mu}+\partial_{\nu} X_{\alpha}^{\mu \nu}$ and $g t_{\alpha}^{\prime \mu} \equiv g_{E} t_{\alpha}^{\mu}+\partial_{\nu} X_{\alpha}^{\mu \nu}$ replacing the unprimed quantities in (3) and (4). Other authors obtained somewhat different splits with quantities of different density. Most noteworthy of these is the "Landau-Lifshitz split," which has the form [11]:

$$
g^{2} G^{\mu \nu}=\kappa\left({ }_{L} \mathcal{T}^{\mu \nu}-g^{2}{ }_{L} t^{\mu \nu}\right)=\kappa g^{2} T^{\mu \nu} .
$$

This split yields a symmetric gravitational energymomentum pseudotensor ${ }_{L} t^{\mu \nu}$.

The most thorough study of gravitational energymomentum laws was carried out by Møller [12], who refered to the various alternatives as the energymomentum complex. He argued that components of the metric tensor $g_{\mu \nu}$ may not be the best choice for gravitational field variables; instead, components of an 
(orthonormal) tetrad field are more suitable, because they are essential for incorporating the Dirac equation into the theory. This enabled him to define a split of the form (2) with a new expression for $t_{\alpha}^{\mu}$ that is a true tensor. Let's call it the Møller split. After carefully evaluating its properties and explaining how it resolves the problematic aspects of alternatives, he declared himself satisfied with the result. His result has scarcely been noticed in the literature, but we shall see that there is much to recommend it when formulated in STA gauge theory.

Møller noted, however, that his gravitational energymomentum tensor, though covariant, is still not unique, because its values can be changed by local Lorentz rotations (gauge transformations) of the tetrad field. He investigated ways to make it unique with supplementary conditions, but wisely did not insist on any of them. Instead he concluded that energy in the gravitational field is not localizable, that is, no exact physical meaning can ascribed to "energy content in a finite part of space." Einstein came to the same conclusion about his pseudotensor, which he maintained throughout his life as the best available descriptor of gravitational energymomentum.

The view that gravitational energymomentum and angular momentum conservation laws are well-defined only asymptotically seems to be the general consensus of workers in GR today [8,9], though they recognize that this entails problematic ambiguities in the detection of gravitational waves. Still, there are dissenters who argue that the ambiguities can be removed by regarding GR as field theory on (flat) Minkowski spacetime. In particular, GR has been developed as the field theory of a spin-2 particle, the graviton $[13,14,15]$. It is noteworthy that generating a self-consistent gravitational energymomentum tensor is a key ingredient of that approach.

On quite a different note, Rosen observed that physicists working with GR invariably have a flat space background in mind, so he elevated the existence of a flat background to an axiom in his Bimetric general relativity [16]. Later it was noted that introducing a flat background metric is mathematically equivalent to the tetrad formulation of GR [17], though it appears to be quite different conceptually. Indeed, Rosen was most interested in using his Bimetric theory to generate alternatives to Einstein's equation (1), especially to eliminate Black Hole singularities.

Introducing a Minkowski background into GR does not alter its mathematical content, as is evident in the gauge theory formulation below. Moreover, it is essential for energymomentum conservation as a consequence of Poincaré invariance in special relativity, with its unparalleled success in relativistic quantum theory. Indeed, Babak and Grishchuk argue that "the Minkowiski metric is not an artificially imposed "prior geometry" but a reflection of experimental facts," and they employ it to derive a unique gravitational energymomentum tensor [18] without the deficiences of standard pseudotensors. Though differing in detail, the present approach supports their general conclusions.

In preparation for the main event, Section II reviews the necessary basics of spacetime algebra, while Sections III and IV summarize essentials of gauge theory gravity and its relation to general relativity. In Section IV Einstein's 
gravitational field tensor is given a simple new "unitary" formulation directly in terms of the curvature tensor without the usual reference to the Ricci tensor. This greatly simplifies the formulation and analysis of the classic energymomentum complexes, as demonstrated in Section V. Then, the main event in Section VI presents a stunning simplification in the formulation and analysis of the energymomentum complex that opens up many new possibilities. This new canonical energymomentum complex passes an initial test for physical relevance in Section VII.

\section{Spacetime Algebra Basics}

This section summarizes basic STA definitions and algebraic identities to be used in this paper. Further results will be introduced as needed. More details and broader discussion are given in the references $[1,2,19,20]$.

For physicists familiar with the Dirac matrix algebra, the quickest approach to the STA is by reinterpreting the Dirac matrices a righthanded orthonormal basis $\left\{\gamma_{\mu} ; \mu=0,1,2,3\right\}$ for a $4 \mathrm{D}$ real Minkowski vector space $\mathcal{V}^{4}$ with signature specified by the rules:

$$
\gamma_{0}^{2}=1 \quad \text { and } \quad \gamma_{1}^{2}=\gamma_{2}^{2}=\gamma_{3}^{2}=-1
$$

Note that the scalar 1 in these equations would be replaced by the identity matrix if the $\gamma_{\mu}$ were Dirac matrices. Thus, (6) is no mere shorthand for matrix equations but a defining relation of vectors to scalars that encodes spacetime signature in algebraic form.

The frame $\left\{\gamma_{\mu}\right\}$ generates an associative geometric algebra that is isomorphic to the Dirac algebra. The product $\gamma_{\mu} \gamma_{\nu}$ of two vectors is called the geometric product. The usual inner product of vectors is defined by

$$
\gamma_{\mu} \cdot \gamma_{\nu} \equiv \frac{1}{2}\left(\gamma_{\mu} \gamma_{\nu}+\gamma_{\nu} \gamma_{\mu}\right)=\eta_{\mu} \delta_{\mu \nu}
$$

where $\eta_{\mu}=\gamma_{\mu}^{2}$ is the signature indicator. The outer product

$$
\gamma_{\mu} \wedge \gamma_{\nu} \equiv \frac{1}{2}\left(\gamma_{\mu} \gamma_{\nu}-\gamma_{\nu} \gamma_{\mu}\right)=-\gamma_{\nu} \wedge \gamma_{\mu}
$$

defines a new entity called a bivector (or 2-vector), which can be interpreted as a directed plane segment representing the plane containing the two vectors.

STA is the real geometric algebra $\mathcal{G}_{4}=\mathcal{G}\left(\mathcal{V}^{4}\right)$ generated by $\mathcal{V}^{4}$ with real scalars. A full basis for the algebra is given by the set:

$\begin{array}{lllll}1 & \left\{\gamma_{\mu}\right\} & \left\{\gamma_{\mu} \wedge \gamma_{\nu}\right\} & \left\{\gamma_{\mu} i\right\} & i \\ 1 \text { scalar } & 4 \text { vectors } & 6 \text { bivectors } & 4 \text { trivectors } & 1 \text { pseudoscalar } \\ \text { grade 0 } & \text { grade 1 } & \text { grade 2 } & \text { grade } 3 & \text { grade } 4\end{array}$

where the righthanded unit pseudoscalar

$$
i \equiv \gamma_{0} \gamma_{1} \gamma_{2} \gamma_{3}
$$


squares to -1 , anticommutes with all odd grade elements and commutes with even grade elements. Thus, $\mathcal{G}_{4}$ is a graded linear space of dimension $1+4+6+$ $4+1=2^{3}=16$, and any element $M$ can be expressed as a linear combination of the 16 basis elements.

A generic element of the STA is called a multivector. Any multivector $M$ can be expanded into the in the graded forms

$$
M=\alpha+a+F+b i+\beta i=\sum_{k=0}^{4}\langle M\rangle_{k},
$$

where $\alpha=\langle M\rangle_{0}$ and $\beta$ are scalars, $a=\langle M\rangle_{1}$ and $b$ are vectors, and $F=\langle M\rangle_{2}$ is a bivector, while $b i=\langle M\rangle_{3}$ is a trivector (or pseudovector) and $\beta i=\langle M\rangle_{4}$ is a pseudoscalar. It is often convenient to drop the subscript on the scalar part so $\langle M\rangle=\langle M\rangle_{0}$. The scalar part behaves like the "trace" in matrix algebra; for example, $\langle M N\rangle=\langle N M\rangle$ for arbitrary multivectors $M$ and $N$. A multivector is said to be even if the grades of its nonvanishing components are all even. The even multivectors compose the even subalgebra of $\mathcal{G}_{4}$, which is, of course, closed under the geometric product.

Coordinate-free computations are facilitated by various definitions. The operation of reversion reverses the order in a product of vectors, so for vectors $a, b, c$ it is defined by

$$
(a b c)^{\sim}=c b a
$$

It follows for any multivector $M$ in the graded form (10) that the reverse $\widetilde{M}$ is given by

$$
\widetilde{M}=\alpha+a-F-b i+\beta i \text {. }
$$

Computations are also facilitated by defining various products in terms of the fundamental geometric product.

The inner and outer products of vectors defined by (7) and (8) can be generalized to arbitrary multivectors as follows. For $A=\langle A\rangle_{r}$ and $B=\langle B\rangle_{s}$ of grades $r, s \geq 0$, inner and outer products can be defined by

$$
A \cdot B \equiv\langle A B\rangle_{|r-s|}, \quad A \wedge B \equiv\langle A B\rangle_{r+s} .
$$

This enables a remarkably simple realization for the classical mathematical concept of duality. The dual of any multivector is obtained by multiplying it by the pseudoscalar $i$ (or some scalar multiple thereof). Thus, in (10) the trivector $b i$ is the dual of the vector $b$. Inner and outer products are related by the duality identities

$$
a \cdot(B i)=(a \wedge B) i, \quad a \wedge(B i)=(a \cdot B) i
$$

Coordinate-free manipulations are facilitated by a system of identities involving inner and outer products $[4,5]$. These identities generalize to arbitrary 
dimensions the well-known identities for dot and cross products in ordinary $3 \mathrm{D}$ vector algebra. We will need the following identities. For a vector $a$, the geometric product is related to inner and outer products by

$$
a B=a \cdot B+a \wedge B .
$$

For vectors $a, b, c$ the most commonly used identity is

$$
a \cdot(b \wedge c)=(a \cdot b) c-(a \cdot c) b=a \cdot b c-a \cdot c b,
$$

where parentheses have been dropped with the understanding that inner products have precedence in ambiguous expressions. The expansion (16) is a special case of

$$
a \cdot(b \wedge B)=a \cdot b B-b \wedge(a \cdot B)
$$

for $\operatorname{grade}(B) \geq 2$, and we have a related identity

$$
a \cdot(b \cdot B)=(a \wedge b) \cdot B .
$$

Inner and outer products are very helpful for defining and manipulating relations among vectors. For example, the entire theory of determinants can be efficiently developed along the following lines [5]. Let $\left\{a_{j} ; j=1,2, \ldots, m\right\}$ and $\left\{b_{k} ; k=1,2, \ldots, m\right\}$ be sets of $m$ vectors referred to "row vectors" and "column vectors" for convenience. The $m \times m$ determinant of the matrix $\left\{a_{j k} \equiv a_{j} \cdot b_{k}\right\}$ is defined by

$$
\operatorname{det}\left(a_{j k}\right)=\operatorname{det}\left(a_{j} \cdot a_{k}\right)=\left(a_{m} \wedge \ldots \wedge a_{2} \wedge a_{1}\right) \cdot\left(b_{1} \wedge b_{2} \wedge \ldots \wedge b_{m}\right) .
$$

Note how the outer product gives the antisymmetry of the determinant underchange of any pair of rows or columns, and the inner product gives symmetry under interchange of all rows with all columns. The standard Laplace expansion for a determinant is an application of the rules (18) and (17); thus, the expansion with respect to the first row of the determinant specified by vector $a_{1}$ is

$$
\begin{aligned}
\operatorname{det}\left(a_{j k}\right)= & \left(a_{m} \wedge \ldots \wedge a_{2}\right) \cdot\left[a_{1} \cdot\left(b_{1} \wedge b_{2} \wedge \ldots \wedge b_{m}\right)\right] \\
= & a_{11}\left(a_{m} \wedge \ldots \wedge a_{2}\right) \cdot\left(b_{2} \wedge \ldots \wedge b_{m}\right) \\
& \quad-a_{12}\left(a_{m} \wedge \ldots \wedge a_{2}\right) \cdot\left(b_{1} \wedge b_{3} \wedge \ldots \wedge b_{m}\right)+a_{13} \ldots .
\end{aligned}
$$

Now, let $\left\{g_{\mu}\right\}$ be a righthanded frame of vectors spanning $\mathcal{V}^{4}$, so its pseudoscalar is an oriented 4 -volume element proportional to the unit pseudoscalar $i$, that is

$$
g_{0} \wedge g_{1} \wedge g_{2} \wedge g_{3}=g i
$$

In terms of the metric tensor for the frame $g_{\mu \nu} \equiv g_{\mu} \cdot g_{\nu}$, the magnitude $g$ of the volume is given by the determinant

$$
\begin{aligned}
-g^{2} & =\left(g_{0} \wedge g_{1} \wedge g_{2} \wedge g_{3}\right)^{2}=\operatorname{det}\left(g_{\mu \nu}\right) \\
& =\left(g_{3} \wedge g_{2} \wedge g_{1} \wedge g_{0}\right) \cdot\left(g_{0} \wedge g_{1} \wedge g_{2} \wedge g_{3}\right) .
\end{aligned}
$$


This can be evaluated in terms of the $g_{\mu \nu}$ by the Laplace expansion (20).

Algebraic manipulations with the frame $\left\{g_{\mu}\right\}$ are greatly facilitated by introducing its reciprocal frame $\left\{g^{\mu}\right\}$ defined by the conditions

$$
g^{\mu} \cdot g_{\nu}=\gamma^{\mu} \cdot \gamma_{\nu}=\delta_{\nu}^{\mu} .
$$

These equations have the explicit solution

$$
\begin{aligned}
g^{\mu} & =(-1)^{\mu}\left(g_{0} \wedge \ldots \wedge \stackrel{\vee}{g}_{\mu} \wedge \ldots \wedge g_{3}\right)\left(g_{0} \wedge g_{1} \wedge g_{2} \wedge g_{3}\right)^{-1} \\
& =(-1)^{\mu+1}\left(g_{0} \wedge \ldots \wedge \stackrel{\vee}{g}_{\mu} \wedge \ldots \wedge g_{3}\right) \cdot\left(g_{0} \wedge g_{1} \wedge g_{2} \wedge g_{3}\right) g^{-2}
\end{aligned}
$$

where $\stackrel{v}{g}_{\mu}$ indicates that $g_{\mu}$ is omitted from the product. With the definition $g^{\mu \nu} \equiv g^{\mu} \cdot g^{\nu}$ for the reciprocal metric tensor, we can adopt the usual tensor algebra conventions for raising and lowering tensor indices and summing over repeated upper and lower index pairs.

Besides inner and outer products, we need the commutator product

$$
A \times B \equiv \frac{1}{2}(A B-B A) .
$$

This is especially useful when A is a bivector. Then we have the identity

$$
A B=A \cdot B+A \times B+A \wedge B
$$

which should be compared with (15). It is important to note that for bivector $A$ the commutator product is "grade-preserving" and acts as a "derivation" on geometric products; that is, for any multivectors $M$ and $N$, we have

$$
A \times\langle M\rangle_{k}=\langle A \times M\rangle_{k}
$$

and

$$
A \times(M N)=(A \times M) N+M(A \times N) .
$$

A considerable advantage of STA is enabling a coordinate-free treatment of Lorentz transformations without matrices [19]. Lorentz transformations continuously connected to the identity are called Lorentz rotations. A Lorentz rotation of any multivector $M$ has explicit algebraic representation in the canonical form

$$
M \quad \rightarrow \quad M^{\prime}=L M \widetilde{L},
$$

where $L$ is even multivector called a rotor, which is subject to the normalization condition

$$
L \widetilde{L}=1
$$

It is obvious from (29) that representation of a Lorentz rotation by a rotor is double-valued, though the ambiguity in sign is seldom an issue. 


\section{Gauge Gravity with Coordinate Frames}

Our flat space model of spacetime $\mathcal{M}^{4}=\{x\}$ represents each spacetime point as a vector $x$ in the Minkowski vector space of special relativity [19]. Let $x=$ $x\left(x^{0}, x^{1}, x^{2}, x^{3}\right)$ be a parametrization of the points, in some spacetime region, by an arbitrary set of coordinates $\left\{x^{\mu}\right\}$. Partial derivatives then give a coordinate frame of tangent vectors to the coordinate curves:

$$
e_{\mu} \equiv \partial_{\mu} x=\frac{\partial x}{\partial x^{\mu}}
$$

The inverse of $x=x\left(x^{0}, x^{1}, x^{2}, x^{3}\right)$ is a set of coordinate functions $x^{\mu}=x^{\mu}(x)$ determining a coordinate frame of gradient vectors

$$
e^{\mu}=\nabla x^{\mu}
$$

reciprocal to the frame $\left\{e_{\mu}\right\}$, as expressed by

$$
e_{\mu} \cdot e^{\nu}=e_{\mu} \cdot \nabla x^{\nu}=\partial_{\mu} x^{\nu}=\delta_{\mu}^{\nu} .
$$

In these terms, the gradient operator $\nabla=\partial_{x}$, better known as the vector derivative by the spacetime point $x$ [19], can be defined by the operator equation

$$
\nabla=e^{\mu} \partial_{\mu}
$$

Flat spacetime is characterized by the existence of preferred coordinate systems called inertial coordinates, for which the coordinate frame is an orthonormal set of constant vectors $\gamma_{\mu}=\partial_{\mu} x$, so the coordinate functions are given by the simple functions

$$
x=x^{\mu} \gamma_{\mu} \quad \text { and } \quad x^{\mu}=x \cdot \gamma^{\mu} .
$$

Inertial systems are related by Poincaré transformations of the general form

$$
x \rightarrow x^{\prime}=L x \widetilde{L}+c,
$$

where $L$ is a constant rotor. The corresponding inertial frames are then related by the Lorentz rotation:

$$
\gamma_{\mu}^{\prime}=L \gamma_{\mu} \widetilde{L}
$$

Gauge theory gravity (GTG) is based on two general principles: Position Gauge Invariance and Local Rotation Covariance, which can be regarded as refinements of Einstein's principles of general relativity and equivalence [?]. The first principle is satisfied by introducing gravity as a gauge tensor field $\bar{h}(a)=\bar{h}(a ; x)$, which is a position-dependent linear function on vector fields, and the dependence on $x$ is usually left implicit. In particular, the gauge tensor $\bar{h}$ maps the coordinate frame $e^{\mu}$ into a corresponding gravity frame

$$
g^{\mu}=\bar{h}\left(e^{\mu}\right)=\bar{h}\left(\nabla x^{\mu}\right) .
$$


Its reciprocal frame is then

$$
g_{\mu}=\underline{h}^{-1}\left(e_{\mu}\right),
$$

where the linear operator $\underline{h}$ is the transpose of $\bar{h}$.

Either frame $\left\{g^{\mu}\right\}$ or $\left\{\bar{g}_{\mu}\right\}$ can be regarded as representing the gravitational field. Indeed, the inner products

$$
g_{\mu \nu}=g_{\mu} \cdot g_{\nu}=\underline{h}^{-1}\left(e_{\mu}\right) \cdot \underline{h}^{-1}\left(e_{\nu}\right)=e_{\mu} \cdot\left[\bar{h}^{-1} \underline{h}^{-1}\left(e_{\nu}\right)\right] .
$$

are precisely equivalent to the components of the metric tensor in GR. Whence the displacement differential $d x=d x^{\mu} e_{\mu}$ generates the familiar line element

$$
d \tau^{2}=g_{\mu \nu} d x^{\mu} d x^{\nu}
$$

in the tensor formulation of GR.

For an inertial frame the pseudoscalar is determined solely by the gauge tensor, thus

$$
-g^{0} \wedge g^{1} \wedge g^{2} \wedge g^{3}=\bar{h}\left(\gamma_{0}\right) \wedge \bar{h}\left(\gamma_{1}\right) \wedge \bar{h}\left(\gamma_{2}\right) \wedge \bar{h}\left(\gamma_{3}\right)=\operatorname{det}(\bar{h}) i .
$$

Writing $h \equiv \operatorname{det}(\bar{h})$, we find from (22) that the squared volume of an arbitrary frame is given by

$$
\operatorname{det}\left(g_{\mu \nu}\right)=\operatorname{det}\left(g_{\mu} \cdot g_{\nu}\right)=h^{-2} \operatorname{det}\left(e_{\mu} \cdot e_{\nu}\right) .
$$

Thus, the ratio $h^{-1}$ of the volume elements for the two metric tensors is a gravitational invariant independent of the coordinate system. This quantity plays a prominent role in Rosen's Bimetric Theory [16].

The second GTG principle is satisfied by introducing a "gauge covariant derivative" or coderivative, with components $D_{\mu}$ defined, for operating on a multivector field $M=M(x)$, by

$$
D_{\mu} M=\partial_{\mu} M+\omega_{\mu} \times M,
$$

where the connexion for the coderivative

$$
\omega_{\mu}=\omega\left(g_{\mu}\right)
$$

is a bivector-valued tensor evaluated on the frame $\left\{g_{\mu}\right\}$. It follows from the bivector properties (27) and (28) that the coderivative $D_{\mu}$ has the same algebraic properties as the operator $\partial_{\mu}$. Note that (27) implies that

$$
D_{\mu}\langle M\rangle=\partial_{\mu}\langle M\rangle \text {. }
$$

In other words, $D_{\mu}=\partial_{\mu}$ when operating on scalar-valued functions.

To maintain form invariance of the coderivative under local Lorentz rotations of the form (29) generated by a position dependent rotor $L=L(x)$, that is, to ensure the transformation

$$
D_{\mu} M \quad \rightarrow \quad L\left(D_{\mu} M\right) \widetilde{L}=D_{\mu}^{\prime} M^{\prime}=\partial_{\mu} M^{\prime}+\omega_{\mu}^{\prime} \times M^{\prime},
$$


the connexion must obey the transformation law

$$
\omega_{\mu}=\rightarrow \quad \omega_{\mu}^{\prime}=L \omega_{\mu}^{\prime} \widetilde{L}-2\left(\partial_{\mu} L\right) \widetilde{L}
$$

A coordinate-free vector coderivative can be defined by the operator equation

$$
D=g^{\mu} D_{\mu},
$$

from which the directional coderivatives $D_{\mu}=g_{\mu} \cdot D$ can be recovered.

For the gravity frame $\left\{g_{\mu}\right\}$ the coderivative (44) takes the form

$$
D_{\mu} g_{\nu}=\partial_{\mu} g_{\nu}+\omega_{\mu} \cdot g_{\nu} .
$$

Note that under coordinate transformations the connexion $\omega_{\mu}$ is a (covariant) tensor, whereas $D_{\mu} g_{\nu}$ is a pseudotensor. Explicit transformation laws induced by a change of coordinates from $x^{\mu}$ to $x^{\prime \mu}$ follow from the chain rule for partial differentiation:

$$
\partial_{\mu}=a_{\mu}^{\nu} \partial_{\nu}^{\prime} \quad \text { with } \quad a_{\mu}^{\nu}=\frac{\partial x^{\mu}}{\partial x^{\prime \mu}}
$$

Hence, from (31) and (39) we get

$$
g_{\mu}=a_{\mu}^{\nu} g_{\nu}^{\prime} \quad \text { and } \quad \omega_{\mu}=\omega\left(a_{\mu}^{\nu} g_{\nu}^{\prime}\right)=a_{\mu}^{\nu} \omega_{\nu}^{\prime} .
$$

Whence, from (50) we obtain the pseudotensor transformation law

$$
D_{\mu} g_{\nu}=a_{\mu}^{\alpha} a_{\nu}^{\beta} D_{\mu}^{\prime} g_{\nu}^{\prime}+a_{\mu}^{\alpha}\left(\partial_{\alpha}^{\prime} a_{\nu}^{\beta}\right) g_{\beta}^{\prime} \text {. }
$$

To relate (50) to the standard covariant derivative in GR, we write

$$
D_{\mu} g_{\nu}=L_{\mu \nu}^{\alpha} g_{\alpha}
$$

where the $L_{\mu \nu}^{\alpha}=g^{\alpha} \cdot\left(D_{\mu} g_{\nu}\right)$ are the usual coefficients of connexion. And differentiating (23) we get

$$
D_{\mu} g^{\alpha}=-L_{\mu \nu}^{\alpha} g^{\nu}
$$

From (44) and (46) it follows that

$$
\partial_{\mu} g_{\alpha \beta}=D_{\mu} g_{\alpha \beta}=\left(D_{\mu} g_{\alpha}\right) \cdot g_{\beta}+g_{\alpha} \cdot\left(D_{\mu} g_{\beta}\right)=L_{\mu \alpha}^{\nu} g_{\nu \beta}+g_{\alpha \nu} L_{\mu \beta}^{\nu},
$$

which is the usual condition for "metric compatibility" of the connexion.

To make the connexion purely "metric based," we need to impose the condition for vanishing torsion:

$$
D \wedge g^{\mu}=D \wedge D x^{\mu}=0
$$

It follows that

$$
D \wedge g^{\mu}=g^{\alpha} \wedge D_{\alpha} g^{\mu}=g^{\beta} \wedge g^{\alpha} L_{\alpha \beta}^{\mu}=0
$$


implies $L_{\alpha \beta}^{\mu}=L_{\beta \alpha}^{\mu}$, or equivalently,

$$
D_{\alpha} g_{\beta}=D_{\beta} g_{\alpha} .
$$

This symmetry condition suffices to solve the equations (54) to get

$$
L_{\mu \nu}^{\alpha}=\frac{1}{2} g^{\alpha \beta}\left(\partial_{\mu} g_{\nu \beta}+\partial_{\nu} g_{\mu \beta}-\partial_{\beta} g_{\mu \nu}\right),
$$

which will be recognized as the Christoffel form for the connexion in GR. This completes our demonstration of equivalence between GTG and GR.

To facilitate subsequent computations, we record a number of useful identities that follow from the above relations. From (50) we get

$$
2 \omega_{\mu}=g^{\nu} \wedge \partial_{\mu} g_{\nu}-g^{\nu} \wedge D_{\mu} g_{\nu},
$$

which can be put in several alternative forms by differentiating $g^{\nu} \wedge g^{\alpha} g_{\nu \alpha}=0$ to get

$$
g^{\nu} \wedge \partial_{\mu} g_{\nu}=g_{\nu} \wedge \partial_{\mu} g^{\nu}=g^{\nu} \wedge g^{\alpha} g_{\nu} \cdot \partial_{\mu} g_{\alpha},
$$

and using (57) to get

$$
g^{\nu} \wedge D_{\mu} g_{\nu}=D \wedge g_{\mu}=\left(D g_{\nu \mu}\right) \wedge g^{\nu} .
$$

Contraction of (54) gives

$$
g^{\nu} \cdot\left(D_{\mu} g_{\nu}\right)=L_{\mu \nu}^{\nu}=\frac{1}{2} g^{\alpha \beta} \partial_{\mu} g_{\alpha \beta}=\partial_{\mu} \ln g=D \cdot g_{\nu} .
$$

Whence

$$
D \cdot\left(g^{-1} g_{\nu}\right)=0 \text {. }
$$

In a similar way we obtain

$$
D \cdot g^{\mu}=-g^{\alpha \beta} L_{\alpha \beta}^{\mu}=\partial_{\nu}\left(g g^{\mu \nu}\right) .
$$

Contraction of (50) gives

$$
D_{\mu} g^{\mu}=-L_{\mu \mu}^{\mu} g^{\nu}=-D \ln g=\partial_{\mu} g^{\mu}+\omega_{\mu} \cdot g^{\mu} .
$$

Whence

$$
D_{\mu}\left(g g^{\mu}\right)=0,
$$

and

$$
g^{\mu} \cdot \omega_{\mu}=\partial_{\mu} g^{\mu}+D \ln g=\partial_{\mu}\left(g g^{\mu}\right) .
$$

Experts will recognize that the frame vectors $g^{\mu}$ are equivalent to the usual tetrad variables $h_{(\alpha)}^{\mu}$, as expressed by

$$
h_{(\alpha)}^{\mu}=g^{\mu} \cdot \gamma_{(\alpha)},
$$


where the lower index is placed in parenthesis to indicate that it is not (necessarily) related to the coordinates. Thus, the STA formalism developed here can be regarded as just another way - perhaps a more elegant, transparent and efficient way - to treat GR in terms of tetrad variables. However, gauge theory offers a deeper theoretical advantage, which is manifested by inserting (38) into (70) to get

$$
h_{(\alpha)}^{\mu}=\bar{h}\left(e^{\mu}\right) \cdot \gamma_{(\alpha)}=e^{\mu} \cdot \underline{h}\left(\gamma_{(\alpha)}\right)=\underline{h}\left(\gamma_{(\alpha)}\right) \cdot \nabla x^{\mu},
$$

This provides an explicit separation of tetrad dependence on arbitrary coordinates $x^{\mu}$ from its dependence on the gravitational gauge tensor $\bar{h}$. It is the critical difference between GTG and standard tetrad theory of GR.

\section{Curvature and Field Equations}

The commutator of coderivatives defined by (44) gives us

$$
\left[D_{\mu}, D_{\nu}\right] M=R\left(g_{\mu} \wedge g_{\nu}\right) \times M
$$

where

$$
R\left(g_{\mu} \wedge g_{\nu}\right)=\partial_{\mu} \omega_{\nu}-\partial_{\nu} \omega_{\mu}+\omega_{\mu} \times \omega_{\nu}
$$

is the curvature tensor expressed as a bivector-valued function of a bivector variable. Contraction of the curvature tensor gives the Ricci tensor

$$
R\left(g_{\nu}\right)=g^{\mu} \cdot R\left(g_{\mu} \wedge g_{\nu}\right),
$$

and a second contraction gives the scalar curvature

$$
R=g^{\nu} \cdot R\left(g_{\nu}\right)=\left(g^{\nu} \wedge g^{\mu}\right) \cdot R\left(g_{\mu} \wedge g_{\nu}\right) .
$$

Einstein's gravitational field equation can thus be written in the form

$$
G\left(g_{\nu}\right) \equiv R\left(g_{\nu}\right)-\frac{1}{2} g_{\nu} R=\kappa T\left(g_{\nu}\right) .
$$

Of course, decomposing this into tensor components $G_{\mu \nu}=g_{\mu} \cdot G\left(g_{\nu}\right)$ and $T_{\mu \nu}=$ $g_{\mu} \cdot T\left(g_{\nu}\right)$ yields Einstein's equation in its standard tensor component form (1).

In this paper we are interested only in equations for the gravity field variables $g_{\mu}$, so we write (72) in the form

$$
\left[D_{\mu}, D_{\nu}\right] g^{\alpha}=\omega_{\mu \nu} \cdot g^{\alpha}=R_{\mu \nu \beta}^{\alpha} g^{\beta},
$$

where

$$
\omega_{\mu \nu} \equiv R\left(g_{\mu} \wedge g_{\nu}\right)=D_{\mu} \omega_{\nu}-D_{\nu} \omega_{\mu}-\omega_{\mu} \times \omega_{\nu},
$$

and, to make contact with the standard tensor formalism, we use (54) to write

$$
R_{\mu \nu \beta}^{\alpha}=\omega_{\mu \nu} \cdot\left(g_{\beta} \wedge g^{\alpha}\right)=\partial_{\mu} L_{\nu \beta}^{\alpha}-\partial_{\nu} L_{\mu \beta}^{\alpha}+L_{\nu \beta}^{\lambda} L_{\mu \lambda}^{\alpha}-L_{\mu \beta}^{\lambda} L_{\nu \lambda}^{\alpha} .
$$


We will also make use of the curvature bivector expressed directly in terms of the coderivative:

$$
\omega_{\mu \nu}=-\frac{1}{2} g_{\alpha} \wedge\left[D_{\mu}, D_{\nu}\right] g^{\alpha}=-\frac{1}{2} g^{\alpha} \wedge\left[D_{\mu}, D_{\nu}\right] g_{\alpha} .
$$

At last we are prepared to formulate the central new contribution of this paper. STA enables us to express Einstein's tensor $G^{\beta}=G\left(g^{\beta}\right)$ in the new unitary form:

$$
G^{\beta}=\frac{1}{2}\left(g^{\beta} \wedge g^{\mu} \wedge g^{\nu}\right) \cdot \omega_{\mu \nu}=\frac{1}{2}\left(g^{\beta} \wedge \omega_{\mu \nu}\right) \cdot\left(g^{\mu} \wedge g^{\nu}\right) .
$$

The last equality is a consequence of the well-known symmetry of the curvature tensor,

$$
\left(g_{\alpha} \wedge g_{\beta}\right) \cdot R\left(g_{\mu} \wedge g_{\nu}\right)=\left(g_{\mu} \wedge g_{\nu}\right) \cdot R\left(g_{\alpha} \wedge g_{\beta}\right)
$$

Using (17) and (18) to expand the inner product in (81), we get

$$
G^{\beta}=g^{\mu} \cdot \omega_{\mu \nu} g^{\nu \beta}-\frac{1}{2} g^{\beta}\left(g^{\nu} \wedge g^{\mu}\right) \cdot \omega_{\mu \nu},
$$

which will be recognized as equivalent to the standard form (76) for the Einstein tensor. Thus, expansion of the inner product has split the "unitary" Einstein tensor into two parts. Let us refer to this as the Ricci split of the Einstein tensor. We shall see that there are alternative splits that may be more physically significant.

As is well-known, the Bianchi identity implies that the codivergence of the Einstein tensor vanishes, that is

$$
\grave{G}(\grave{D})=\frac{1}{2}\left(g^{\mu} \wedge g^{\nu} \wedge D\right) \cdot \omega_{\mu \nu}=\frac{1}{2}\left(g^{\beta} \wedge g^{\mu} \wedge g^{\nu}\right) \cdot\left(D_{\beta} \omega_{\mu \nu}\right)=0,
$$

where the implication $D \wedge\left(g^{\mu} \wedge g^{\nu}\right)=0$ from (57) has been used. From the antisymmetry of the outer products, it follows that the Bianchi identity can be written in the instructive form

$$
D_{\alpha} \omega_{\mu \nu}+D_{\mu} \omega_{\nu \alpha}+D_{\nu} \omega_{\alpha \mu}=0 .
$$

With the help of (68) we can write the codivergence of Einstein's tensor in the alternative form

$$
D_{\mu}\left(g G^{\mu}\right)=g \grave{G}(\grave{D})+G\left[D_{\mu}\left(g g^{\mu}\right)\right]=0 .
$$

\section{Energymomentum Splits in General Relativity}

To facilitate comparison with standard GR, we first study the energymomentum tensor in component form. According to (81), the components of Einstein's tensor are given by

$$
G_{\alpha}^{\beta}=G^{\beta} \cdot g_{\alpha}=\frac{1}{2}\left(g^{\beta} \wedge g^{\mu} \wedge g^{\nu}\right) \cdot\left(\omega_{\mu \nu} \wedge g_{\alpha}\right)=g^{\beta} \cdot G_{\alpha},
$$


which shows that it is a symmetrical tensor. With the help of (83), contraction retrieves the scalar curvature:

$$
G_{\alpha}^{\alpha}=G^{\alpha} \cdot g_{\alpha}=\left(g^{\mu} \wedge g^{\nu}\right) \cdot \omega_{\mu \nu}=-R .
$$

Adopting $g R$ as Lagrangian density, Hilbert retrieved Einstein's tensor by a variation with respect to the metric tensor. Einstein put this Lagrangian in an alternative form which we can obtain by inserting (80) to get

$$
\begin{aligned}
g R & =g\left(g^{\nu} \wedge g^{\mu}\right) \cdot\left[g^{\lambda} \wedge D_{\nu} D_{\mu} g_{\lambda}\right] \\
& =g\left(g^{\nu} \wedge g^{\mu}\right) \cdot\left[D_{\nu}\left(g^{\lambda} \wedge D_{\mu} g_{\lambda}\right)-\left(D_{\nu} g^{\lambda}\right) \wedge\left(D_{\mu} g_{\lambda}\right)\right] \\
& =\partial_{\nu} W^{\nu}+\mathcal{L}
\end{aligned}
$$

where $W^{\nu} \equiv g\left(g^{\nu} \wedge g^{\mu}\right) \cdot\left(g^{\lambda} \wedge D_{\mu} g_{\lambda}\right)$ and

$$
\begin{aligned}
\mathcal{L} & =g\left(g^{\nu} \wedge g^{\mu}\right) \cdot\left[-\left(D_{\nu} g^{\lambda}\right) \wedge\left(D_{\mu} g_{\lambda}\right)\right] \\
& =g\left(g^{\nu} \wedge g^{\mu}\right) \cdot\left(g^{\beta} \wedge g_{\alpha}\right) L_{\nu \beta}^{\lambda} L_{\mu \lambda}^{\alpha} \\
& =g g^{\nu \beta}\left(L_{\mu \beta}^{\lambda} L_{\nu \lambda}^{\mu}-L_{\nu \beta}^{\lambda} L_{\mu \lambda}^{\mu}\right) .
\end{aligned}
$$

The last line in (89) follows from

$$
g\left(g^{\nu} \wedge g^{\mu}\right) \cdot\left[D_{\nu}\left(g^{\lambda} \wedge D_{\mu} g_{\lambda}\right)\right]=D_{\nu} W^{\nu}=\partial_{\nu} W^{\nu}
$$

by using the identity

$$
D_{\nu}\left[g\left(g^{\nu} \wedge g^{\mu}\right)\right]=g\left(D \wedge g^{\mu}\right)=0,
$$

which is an easy consequence of (57) and (68).

Einstein observed that $\partial_{\nu} W^{\nu}$ does not contribute to variations on the boundary, so he derived his energymomentum tensor by varying his "effective Lagrangian" $\mathcal{L}$ with respect to the metric tensor [7]. The calculation is rather messy because it requires expressing the $L_{\nu \lambda}^{\mu}$ in (90) in terms of the metric tensor. An easier and more transparent calculation is given below.

Møller got a different result by applying Einstein's approach to his tetrad formulation of the energymomentum complex [12]. We can obtain his result by inserting (78) into (88) to get

$$
\begin{aligned}
g R & =g\left(g^{\nu} \wedge g^{\mu}\right) \cdot\left[2 D_{\mu} \omega_{\nu}+\omega_{\nu} \times \omega_{\mu}\right] \\
& =\partial_{\nu} \hat{W}^{\nu}+\hat{L},
\end{aligned}
$$

where the identity (92) is again used to identify $\hat{W}^{\nu} \equiv 2 g\left(g^{\nu} \wedge g^{\mu}\right) \cdot \omega_{\nu}$ and Møller's "effective Lagrangian" has the various forms

$$
\begin{aligned}
\hat{\mathcal{L}} & =g\left(g^{\nu} \wedge g^{\mu}\right) \cdot\left[\omega_{\nu} \times \omega_{\mu}\right]=g\left\langle\left(g^{\nu} \wedge g^{\mu}\right) \omega_{\nu} \omega_{\mu}\right\rangle \\
& =g\left[\left(g^{\nu} \wedge g^{\mu}\right) \times \omega_{\nu}\right] \cdot \omega_{\mu} .
\end{aligned}
$$


Again, rather that calculating the variation of this Lagrangian with respect to tetrad components as Møller did, we derive the Møller split directly from the Einstein tensor below.

We are now prepared to derive and elucidate the Einstein split (2) of the $g G_{\alpha}^{\beta}$. The derivation begins by inserting (80) into (81) to get Einstein's tensor in the form

$$
G^{\beta}=\frac{1}{2}\left(g^{\beta} \wedge g^{\mu} \wedge g^{\nu}\right) \cdot\left[g^{\lambda} \wedge D_{\nu} D_{\mu} g_{\lambda}\right] .
$$

The split is then easily then immediately given by the following:

$$
\begin{aligned}
g G_{\alpha}^{\beta} & =\frac{1}{2} g\left(g^{\beta} \wedge g^{\mu} \wedge g^{\nu}\right) \cdot\left[g_{\alpha} \wedge g^{\lambda} \wedge D_{\nu} D_{\mu} g_{\lambda}\right] \\
& =\frac{1}{2} g\left(g^{\beta} \wedge g^{\mu} \wedge g^{\nu}\right) \cdot\left[D_{\nu}\left(g_{\alpha} \wedge g^{\lambda} \wedge D_{\mu} g_{\lambda}\right)-D_{\nu}\left(g_{\alpha} \wedge g^{\lambda}\right) \wedge\left(D_{\mu} g_{\lambda}\right)\right] \\
& =\kappa\left[\partial_{\nu} U_{\alpha}^{\beta \nu}-g_{E} t_{\alpha}^{\beta}\right] .
\end{aligned}
$$

where ${ }_{F} U_{\alpha}^{\beta \nu}$ is Freud's superpotential [21], explicitly defined by the determinant

$$
\begin{aligned}
{ }_{F} U_{\alpha}^{\beta \nu} & =\frac{g}{2 \kappa}\left(g^{\beta} \wedge g^{\mu} \wedge g^{\nu}\right) \cdot\left(g_{\alpha} \wedge g^{\lambda} \wedge D_{\lambda} g_{\mu}\right) \\
& =\frac{g}{2 \kappa}\left|\begin{array}{ccc}
\delta_{\alpha}^{\nu} & \delta_{\alpha}^{\mu} & \delta_{\alpha}^{\beta} \\
g^{\nu \lambda} & g^{\mu \lambda} & g^{\beta \lambda} \\
L_{\mu \lambda}^{\nu} & L_{\mu \lambda}^{\mu} & L_{\mu \lambda}^{\beta}
\end{array}\right|
\end{aligned}
$$

The last equality in (96) is a consequence of

$$
\partial_{\nu F} U_{\alpha}^{\beta \nu}=D_{\nu F} U_{\alpha}^{\beta \nu}=\frac{g}{2 \kappa}\left(g^{\beta} \wedge g^{\mu} \wedge g^{\nu}\right) \cdot D_{\nu}\left(g_{\alpha} \wedge g^{\lambda} \wedge D_{\mu} g_{\lambda}\right),
$$

which follows from the identity

$$
D_{\nu}\left[g\left(g^{\beta} \wedge g^{\mu} \wedge g^{\nu}\right)\right]=0 .
$$

Identifying Einstein's gravitational energymomentum density in (96) with

$$
g_{E} t_{\alpha}^{\beta}=\frac{g}{2 \kappa}\left(g^{\beta} \wedge g^{\mu} \wedge g^{\nu}\right) \cdot\left[D_{\nu}\left(g_{\alpha} \wedge g^{\lambda}\right) \wedge\left(D_{\mu} g_{\lambda}\right)\right],
$$

we can put Einstein's split (2) in the more explicit form

$$
{ }_{E} \mathcal{T}_{\alpha}^{\beta}=\partial_{\nu F} U_{\alpha}^{\beta \nu}=g T_{\alpha}^{\beta}+g_{E} t_{\alpha}^{\beta},
$$

from which the total energymomentum conservation law

$$
\partial_{\beta E} \mathcal{T}_{\alpha}^{\beta}=\partial_{\beta} \partial_{\nu F} U_{\alpha}^{\beta \nu}=-\partial_{\beta} \partial_{\nu F} U_{\alpha}^{\nu \beta}=0
$$

follows as an identity.

Alternative forms for Einstein's tensor ${ }_{E} t_{\alpha}^{\beta}$ are most easily derived by using the surprising identity

$$
D_{\alpha}\left[g\left(g^{\beta} \wedge g^{\nu}\right)\right]=g\left(g^{\beta} \wedge g^{\nu} \wedge g^{\mu}\right) \cdot\left(D_{\alpha} g_{\mu}\right),
$$


which is readily verified after it has been discovered. Now equation (100) can be expanded as follows:

$$
\begin{aligned}
2 \kappa g_{E} t_{\alpha}^{\beta} & =g\left(g^{\beta} \wedge g^{\mu} \wedge g^{\nu}\right) \cdot\left[\left(D_{\nu} g_{\alpha}\right) \wedge g^{\lambda} \wedge\left(D_{\mu} g_{\lambda}\right)+g_{\alpha} \wedge\left(D_{\nu} g^{\lambda}\right) \wedge\left(D_{\mu} g_{\lambda}\right)\right] \\
& =D_{\alpha}\left[g\left(g^{\beta} \wedge g^{\mu}\right)\right] \cdot\left(g^{\lambda} \wedge D_{\mu} g_{\lambda}\right)+g\left[\left(g^{\beta} \wedge g^{\mu} \wedge g^{\nu}\right) \cdot g_{\alpha}\right] \cdot\left[\left(D_{\nu} g^{\lambda}\right) \wedge\left(D_{\mu} g_{\lambda}\right)\right] \\
& =D_{\alpha}\left[g\left(g^{\beta} \wedge g^{\mu}\right) \cdot\left(g^{\lambda} \wedge g_{\sigma}\right)\right] L_{\mu \lambda}^{\sigma}+\delta_{\alpha}^{\beta} g\left(g^{\mu} \wedge g^{\nu}\right) \cdot\left[\left(D_{\nu} g^{\lambda}\right) \wedge\left(D_{\mu} g_{\lambda}\right)\right] .
\end{aligned}
$$

Identifying Einstein's Lagrangian (90) in the last term, we can put this result into the form

$$
\begin{aligned}
2 \kappa g_{E} t_{\alpha}^{\beta} & =\partial_{\alpha}\left[g\left(g^{\beta} \wedge g^{\mu}\right) \cdot\left(g^{\lambda} \wedge g_{\sigma}\right)\right] L_{\mu \lambda}^{\sigma}+\delta_{\alpha}^{\beta} \mathcal{L} \\
& =L_{\mu \lambda}^{\beta} \partial_{\alpha}\left(g g^{\mu \lambda}\right)-L_{\mu \lambda}^{\mu} \partial_{\alpha}\left(g g^{\beta \lambda}\right)+\delta_{\alpha}^{\beta} \mathcal{L},
\end{aligned}
$$

This expression for Einstein's gravitational energymomentum tensor was first derived by Møller [12].

Møller also derived the following compact expression for Freud's superpotential:

$$
\begin{aligned}
\kappa_{E} U_{\alpha}^{\beta \nu} & =\frac{1}{2} g^{-1} g_{\alpha \sigma} \partial_{\lambda}\left[g^{2}\left(g^{\nu \sigma} g^{\beta \lambda}-g^{\beta \sigma} g^{\nu \lambda}\right)\right] \\
& =\frac{1}{2} g^{-1} g_{\alpha \sigma} \partial_{\lambda}\left[g^{2}\left(g^{\beta} \wedge g^{\nu}\right) \cdot\left(g^{\sigma} \wedge g^{\lambda}\right)\right] .
\end{aligned}
$$

Here, it is an immediate consequence of using identity (103) in (97).

As Møller observed, an obvious variation of this result is a superpotential of the form

$$
\begin{aligned}
\kappa_{L} U^{\beta \nu \sigma} & =\frac{1}{2} g^{-1} \partial_{\lambda}\left[g^{2}\left(g^{\beta} \wedge g^{\nu}\right) \cdot\left(g^{\sigma} \wedge g^{\lambda}\right)\right] \\
& =\frac{1}{2} g\left(g^{\beta} \wedge g^{\mu} \wedge g^{\nu}\right) \cdot\left(g^{\sigma} \wedge g^{\lambda} \wedge D_{\lambda} g_{\mu}\right)
\end{aligned}
$$

This provides an elegant superpotential for the Landau-Lifshitz split (5), where the total energymomentum tensor can now be put in the form:

$$
{ }_{L} \mathcal{T}^{\beta \sigma}=\partial_{\nu}\left(g_{L} U^{\beta \nu \sigma}\right)=\partial_{\nu} \partial_{\lambda}\left(\varphi^{\beta \nu \sigma \lambda}\right)={ }_{L} \mathcal{T}^{\sigma \beta},
$$

where $\varphi^{\beta \nu \sigma \lambda}=g^{2}\left(g^{\beta} \wedge g^{\nu}\right) \cdot\left(g^{\sigma} \wedge g^{\lambda}\right)$ and the symmetry is obvious. However, we shall not follow up this lead, because the alternatives appear to be more promising.

A major consideration in Einstein's energymomentum analysis was that his effective Lagrangian (90) and hence his gravitational energymomentum tensor (100) do not involve derivatives of the metric tensor of higher order than the first. This was reinforced by the simplicity of his decomposition (89) for the scalar curvature density. However, a few years after Einstein's death, Møller discovered that the alternative Lagrangian (94) has the same advantages as Einstein's Lagrangian and even more to recommend it, as we see next.

We derive the Møller split of Einstein's tensor by inserting (78) into (81) to get

$$
G^{\beta}=\frac{1}{2}\left(g^{\beta} \wedge g^{\mu} \wedge g^{\nu}\right) \cdot\left[2 D_{\mu} \omega_{\nu}-\omega_{\mu} \times \omega_{\nu}\right]
$$


The split is then immediately given by the following:

$$
g G_{\alpha}^{\beta}=g\left(g^{\beta} \wedge g^{\mu} \wedge g^{\nu}\right) \cdot\left[\left(D_{\mu} \omega_{\nu}+\frac{1}{2} \omega_{\nu} \times \omega_{\mu}\right) \wedge g_{\alpha}\right]=\kappa\left[\partial_{\mu M} U_{\alpha}^{\beta \mu}-g_{M} t_{\alpha}^{\beta}\right],
$$

where ${ }_{M} U_{\alpha}^{\beta \mu}$ is dubbed Møller's superpotential [12] and explicitly defined by

$$
{ }_{M} U_{\alpha}^{\beta \mu}=\frac{g}{\kappa}\left(g^{\beta} \wedge g^{\mu} \wedge g^{\nu}\right) \cdot\left(\omega_{\nu} \wedge g_{\alpha}\right)
$$

Møller's own expression for his superpotential is considerably more complicated than this, in part because he did not identify the bivector $\omega_{\nu}$ as its crucial ingredient, and, of course, the felicities of STA were not available to him.

As in Einstein's split, an explicit expression for the total energymomentum tensor is derived from Møller's superpotential; thus,

$$
\partial_{\mu M} U_{\alpha}^{\beta \mu}=D_{\mu M} U_{\alpha}^{\beta \mu}=\frac{g}{\kappa}\left(g^{\beta} \wedge g^{\mu} \wedge g^{\nu}\right) \cdot\left[D_{\mu}\left(\omega_{\nu} \wedge g_{\alpha}\right)\right]
$$

Møller's gravitational energymomentum density in (109) can accordingly be identified with

$$
\begin{aligned}
{ }_{M} t_{\alpha}^{\beta} & =\frac{g}{\kappa}\left(g^{\beta} \wedge g^{\mu} \wedge g^{\nu}\right) \cdot\left[\frac{1}{2}\left(\omega_{\mu} \times \omega_{\nu}\right) \wedge g_{\alpha}+\omega_{\nu} \wedge\left(D_{\mu} g_{\alpha}\right)\right] \\
& ={ }_{M} U_{\alpha}^{\beta}+{ }_{M} U_{\sigma}^{\beta \mu}\left(g^{\sigma} \cdot \partial_{\mu} g_{\alpha}\right),
\end{aligned}
$$

where

$$
{ }_{M} U_{\alpha}^{\beta}=\frac{g}{\kappa}\left(g^{\beta} \wedge g^{\mu} \wedge g^{\nu}\right) \cdot\left[\frac{1}{2}\left(\omega_{\mu} \times \omega_{\nu}\right) \wedge g_{\alpha}+\omega_{\nu} \wedge\left(\omega_{\mu} \cdot g_{\alpha}\right)\right] .
$$

This expression can be expanded into many terms in agreement with Møller's result [12]. However, that will not be done here, as the assorted terms are not physically informative. The result of greatest physical interest here is the simple expression (110) for Møller's superpotential, as its divergence gives Møller's total energymomentum tensor according to (109).

The advantages of Møller's split over Einstein's split should now be evident. We have already mentioned Møller's argument that tetrads are more fundamental than metric tensors, because they are essential for introducing spinors and quantum mechanics into GR. Though he never made use of that fact, he did demonstrate that tetrads enable him to create a new kind of superpotential. Møller emphasized that his superpotential (110) is a covariant tensor, whereas Freud's superpotential (97) is a pseudotensor. Accordingly, he was able to show that his energymomentum tensor had certain advantages over Einstein's. His analysis will not be reviewed here, because GTG introduces a new element that greatly simplifies and clarifies the theory. That is the subject of the next section.

As Møller discovered by direct calculation, Møller and Einstein splits are equivalent for some solutions of Einstein's equation such as Schwarzschild's. A general condition for such equivalence is vanishing of the first term on the right side of (61), and (62) shows that this can be reduced to the equivalent condition $g_{\nu} \cdot \partial_{\mu} g_{\alpha}=g_{\alpha} \cdot \partial_{\mu} g_{\nu}$ 


\section{Canonical Energymomentum Split and Inertial Frames}

In the preceding section the energymomentum complex was analyzed in terms of gravity frames $\left\{g_{\mu}\right\}$ to make direct contact with the literature in general relativity and introduce simplifications afforded by the STA formalism. With that background we can appreciate the ultimate simplification in the following version of an energymomentum split.

The new split is obtained by using (99) to put (108) and Einstein's equation in the form

$$
g G\left(g^{\beta}\right)=\kappa D_{\mu} U^{\beta \mu}-\frac{1}{2} g\left(g^{\beta} \wedge g^{\mu} \wedge g^{\nu}\right) \cdot\left(\omega_{\mu} \times \omega_{\nu}\right)=\kappa g T\left(g^{\beta}\right),
$$

where $U^{\beta \mu}$ is the canonical energymomentum superpotential defined by

$$
\kappa U^{\beta \mu} \equiv g\left(g^{\beta} \wedge g^{\mu} \wedge g^{\nu}\right) \cdot \omega_{\nu},
$$

Next we use (44) to get

$$
D_{\mu} U^{\beta \mu}=\partial_{\mu} U^{\beta \mu}+\omega_{\mu} \times U^{\beta \mu},
$$

and thus put (114) in the form

$$
\kappa^{-1} g G\left(g^{\beta}\right)=\partial_{\mu} U^{\beta \mu}-t^{\beta}=T^{\beta},
$$

where

$$
t^{\beta} \equiv U^{\beta \mu} \times \omega_{\mu}+\frac{1}{2} \kappa^{-1} g\left(g^{\beta} \wedge g^{\mu} \wedge g^{\nu}\right) \cdot\left(\omega_{\mu} \times \omega_{\nu}\right)
$$

is identified as the energymomentum tensor for the graviational field and $T^{\beta} \equiv$ $g T\left(g^{\beta}\right)$. Consequently, the total energymomentum tensor (strictly speaking a "tensor density") is defined by

$$
\mathcal{T}^{\beta} \equiv \partial_{\mu} U^{\beta \mu}=t^{\beta}+T^{\beta},
$$

and we have the energymomentum conservation law:

$$
\partial_{\beta} \mathcal{T}^{\beta}=\partial_{\beta}\left(t^{\beta}+T^{\beta}\right)=0 .
$$

We refer to (119) with the associated definitions (115) and (118) as the canonical energymomentum split. It is essentially equivalent to the Møller split discussed in the previous section. However, its formulation is now so simple and its derivation is so straightforward as to be almost trivial. Consequently, we can expect its physical implications to be more transparent and easier to analyze.

The most striking and perhaps the most profound feature of the canonical split is the simple linear relation (115) between the the superpotential, which determines energymomentum density, and the connexion, which determines grav-

itational force. It is a kind of duality between a vector-valued function of a 
bivector variable $\kappa U^{\beta \mu}$ and a bivector-valued function of a vector variable $\omega_{\sigma}$. The duality relation can be inverted by expanding the right side of (115) to get

$$
\kappa U^{\mu \nu}=g\left(g^{\mu} \wedge g^{\nu} \wedge g^{\sigma}\right) \cdot \omega_{\sigma}=\frac{1}{2} \kappa\left(g^{\mu} \wedge g^{\nu}\right) \cdot I+g\left(g^{\mu} \wedge g^{\nu}\right) \cdot \omega_{\sigma} \gamma^{\sigma}
$$

where

$$
I \equiv \frac{1}{2}\left(g_{\nu} \wedge g_{\mu}\right) \cdot U^{\mu \nu}=g_{\nu}\left(g_{\mu} \cdot U^{\mu \nu}\right)=2 \kappa^{-1} g g^{\sigma} \cdot \omega_{\sigma}
$$

is a "frame-independent" vector field. Later we shall be interested in the related frame-independent trivector field

$$
i H \equiv g_{\nu} \wedge g_{\mu} \wedge U^{\mu \nu}=2 \kappa^{-1} g g^{\sigma} \wedge \omega_{\sigma}=-H i
$$

which has been expressed as the dual of a vector field $H$. Solving (121) for the connexion bivector we obtain

$$
\kappa^{-1} g \omega_{\sigma}=\frac{1}{2}\left(g_{\sigma} \wedge g_{\nu} \wedge g_{\mu}\right) \cdot U^{\mu \nu}+\frac{1}{2} I \wedge g_{\sigma} .
$$

This surprising dual equivalence of the connexion $\omega_{\sigma}$ to the superpotential $\kappa U^{\beta \mu}$ can be regarded as a hitherto unsuspected relation of gravitational force to energymomentum. Accordingly, it can be expected to provide new physical insights, which this paper begins to explore.

Physically, the most important question about the canonical split is the uniqueness of the energymomentum tensor defined by (119). In other words, to what degree is energymomentum localizable? We have already noted that the connexion $\omega_{\mu}=\omega\left(g_{\mu}\right)$ is invariant under the position gauge transformations. Hence, the superpotential and all other elements of the canonical split are gauge invariant. This completely solves Einstein's problem of defining a coordinate independent energymomentum tensor. However, in contrast to the Ricci split, the canonical split is not rotation gauge covariant. According to (48), the connexion transforms as a "pseudotensor" under local rotations. Since the superpotential $U^{\mu \nu}$ is directly proportional to $\omega_{\mu}$ and the energymomentum tensor is a gradient thereof, these quantities depend on the choice of the local rotation gauge, which is not uniquely defined by the theory. Similar considerations led Møller [12] to conclude that gravitational energymomentum is not localizable. However, his conclusion overlooks a crucial point: the duality of gravitational force to local energymomentum density. To make the point explicit, consider the geodesic equation for a particle with invariant velocity $v=\underline{h}^{-1}(\dot{x})$ :

$$
v \cdot D v=\dot{v}+\omega(v) \cdot v=0
$$

For a given gauge, the term $-\omega(v) \cdot v$ can be regarded as the gravitational force on the particle. This force changes with a change in gauge, but the superpotential and energymomentum tensor change in the same way. Therefore, the local relation of force to energymomentum is preserved. In other words, with respect to local energymomentum exchange, all gauges are equivalent. In this sense, energymomentum is localizable whatever gauge is chosen. 
So far, we have not fully exploited the fact that GTG augments GR with the explicit assumption of a flat spacetime background. As we have already noted, that entails the existence of distinguished coordinate systems and frames called inertial coordinates and inertial frames. In others words, for the purpose of spacetime measurement we can set up a distinguished class of ideal observers called inertial observers [19, 1]. In contrast to the spacetime history of an inertial observer, the history of a real observer is "curved" by the ambient gravitational field. However, with suitable physical measurements and theoretical calculations the effect of the gravitational field can be ascertained, so an inertial frame can be determined indirectly. In this respect, GTG is similar to Newtonian mechanics, where an inertial frame cannot be defined physically without identifying the ambient forces.

The remainder of this section is devoted to formulation and analysis of conservation laws from the "viewpoint of the mythical inertial observer." It demonstrates how inertial frames simplify and clarify physical interpretation, especially for angular momentum.

We begin by expressing the connexion as an explicit function of the $\gamma_{\mu}$ :

$$
\omega_{\mu}=\omega\left(g_{\mu}\right)=\omega\left(\underline{h}^{-1}\left(\gamma_{\mu}\right)\right) \equiv \Omega\left(\gamma_{\mu}\right) \equiv \Omega_{\mu} .
$$

Note that, according to (42), the metric density $g$ for inertial coordinates is entirely determined by gauge tensor and given explicitly by $g^{-1}=h=\operatorname{det}(\bar{h})$.

Next we introduce an inertial energymomentum superpotential defined by

$$
\kappa \underline{U}^{\beta \mu}=\kappa \underline{U}\left(\gamma^{\beta} \wedge \gamma^{\mu}\right) \equiv\left(\gamma^{\beta} \wedge \gamma^{\mu} \wedge \gamma^{\nu}\right) \cdot \underline{\Omega}_{\nu},
$$

where the "inertial connexion"

$$
\underline{\Omega}_{\nu} \equiv g \underline{h}\left(\Omega_{\nu}\right)
$$

results from transforming the connexion to the "inertial system." It follows that

$$
U^{\beta \mu}=\bar{h}\left(\underline{U}^{\beta \mu}\right) .
$$

This can be proved by using the definition of $\bar{h}$ as the adjoint of $\underline{h}$ as follows:

$$
\begin{aligned}
U_{\alpha}^{\beta \mu} & \equiv U^{\beta \mu} \cdot g_{\alpha}=g\left(g^{\beta} \wedge g^{\mu} \wedge g^{\nu}\right) \cdot\left(\Omega_{\nu} \wedge g_{\alpha}\right) \\
& =g\left[\bar{h}\left(\gamma^{\beta} \wedge \gamma^{\mu} \wedge \gamma^{\nu}\right)\right] \cdot\left[\Omega_{\nu} \wedge \underline{h}^{-1}\left(\gamma_{\alpha}\right)\right] \\
& =g\left(\gamma^{\beta} \wedge \gamma^{\mu} \wedge \gamma^{\nu}\right) \cdot \underline{h}\left[\Omega_{\nu} \wedge \underline{h}^{-1}\left(\gamma_{\alpha}\right)\right] \\
& =\left(\gamma^{\beta} \wedge \gamma^{\mu} \wedge \gamma^{\nu}\right) \cdot \underline{\Omega}_{\nu} \cdot \gamma_{\alpha}=\underline{U}^{\beta \mu} \cdot \gamma_{\alpha} .
\end{aligned}
$$

Hence

$$
U^{\beta \mu}=U^{\beta \mu} \cdot g_{\alpha} g^{\alpha}=\bar{h}\left(\underline{U}^{\beta \mu}\right) \cdot \gamma_{\alpha} \bar{h}\left(\gamma^{\alpha}\right)=\bar{h}\left(\underline{U}^{\beta \mu}\right)
$$

as advertised. 
In strict analogy to (119), from the inertial superpotential $\underline{U}^{\beta \mu}$ we obtain an inertial energymomentum tensor

$$
\underline{\mathcal{T}}^{\beta} \equiv \partial_{\mu} \underline{U}^{\beta \mu}
$$

The relation between the canonical and inertial energymomentum tensors is obtained by differentiating (129), which gives

$$
\partial_{\mu} U^{\beta \mu}=\bar{h}\left(\partial_{\mu} \underline{U}^{\beta \mu}\right)+U_{\alpha}^{\beta \mu} \partial_{\mu} g^{\alpha} .
$$

Accordingly, the canonical and inertial conservation laws are related by

$$
\partial_{\mu} \mathcal{T}^{\beta}=\bar{h}\left[\partial_{\mu} \underline{\mathcal{T}}^{\beta}\right]=0 .
$$

Of course, the entire canonical conservation law (119) can be recast as "inertial conservation law" $\underline{\mathcal{T}}^{\beta}=\underline{t}^{\beta}+\underline{T}^{\beta}$, but we will not bother with explicit definitions for the "inertial tensors" $\underline{t}^{\beta}$ and $\underline{T}^{\beta}$ as we shall not need them. Rather, we devote the rest of this section to analysing properties of $\underline{\mathcal{T}}^{\beta}$. Also, we continue the practice introduced in the last two paragraphs of denoting inertial quantities with underbars to distinguish them from their canonical counterparts, though it is an abuse of our underbar notation for linear operators.

The energymomentum tensor $\underline{\mathcal{T}}^{\mu}$ is not generally symmetric. Its transpose $\overline{\mathcal{T}}^{\mu}$ is defined by the condition $\gamma^{\mu} \cdot \underline{\mathcal{T}}^{\mu}=\overline{\mathcal{T}}^{\mu} \cdot \gamma^{\nu}$, so from (127) and (129) it has the specific form

$$
\left.\kappa \overline{\mathcal{T}}^{\mu}=\nabla \cdot\left[\gamma^{\sigma} \cdot\left(\underline{\Omega}_{\sigma} \wedge \gamma^{\mu}\right)\right]=\nabla \cdot\left[\underline{\Omega}^{\mu}+\left(\gamma^{\sigma} \cdot \underline{\Omega}_{\sigma}\right) \wedge \gamma^{\mu}\right)\right] .
$$

Thus $\overline{\mathcal{T}}^{\mu}$ can be calculated directly from the connexion without bothering with duality. The antisymmetry $\underline{U}^{\mu \nu}=-\underline{U}^{\nu \mu}$ of the superpotential immediately implies the total energymomentum conservation law in the two equivalent forms

$$
\partial_{\mu} \underline{\mathcal{T}}^{\mu}=0 \Longleftrightarrow \nabla \cdot \overline{\mathcal{T}}^{\mu}=0 .
$$

Of course, this ignores the possibility of source singularities, which will be treated separately.

We start by noting that the trace of the energymomentum tensor is

$$
\operatorname{Tr} \underline{\mathcal{T}}^{\mu} \equiv \gamma_{\mu} \cdot \underline{\mathcal{T}}^{\mu}=\partial_{\nu}\left(\gamma_{\mu} \cdot \underline{U}^{\mu \nu}\right)=\nabla \cdot \underline{I},
$$

where, in analogy with (122),

$$
\underline{I} \equiv \frac{1}{2}\left(\gamma_{\nu} \wedge \gamma_{\mu}\right) \cdot \underline{U}^{\mu \nu}=\gamma_{\nu}\left(\gamma_{\mu} \cdot \underline{U}^{\mu \nu}\right)=2 \kappa^{-1} \gamma^{\sigma} \cdot \underline{\Omega}_{\sigma} .
$$

Anticipating a connection to gravitational mass, let us tag the vector field $\underline{I}$ with the suggestive name inertial vector.

Further physical insight comes from considering angular momentum conservation. To get an angular momentum conservation law, we define a generalized orbital angular momentum tensor $\underline{\mathcal{T}}^{\mu} \wedge x$ at each spacetime point $x=x^{\mu} \gamma_{\mu}$. Noting that

$$
\partial_{\mu}\left(\underline{\mathcal{T}}^{\mu} \wedge x\right)=\underline{\mathcal{T}}^{\mu} \wedge \gamma_{\mu}=\partial_{\nu}\left(\underline{U}^{\mu \nu} \wedge \gamma_{\mu}\right),
$$


we identify $\underline{S}^{\mu} \equiv \underline{U}^{\nu \mu} \wedge \gamma_{\nu}$ as an intrinsic spin angular momentum tensor and define the total angular momentum tensor

$$
\underline{\mathcal{M}}^{\mu}=\partial_{\nu}\left(\underline{U}^{\mu \nu} \wedge x\right)=\underline{\mathcal{T}}^{\mu} \wedge x+\underline{S}^{\mu} .
$$

Thus we have an angular momentum superpotential $\underline{U}^{\mu \nu} \wedge x$, from which angular momentum conservation follows:

$$
\partial_{\mu} \underline{\mathcal{M}}^{\mu}=0 .
$$

It is noteworthy that this simple form for the angular momentum conservation law is unique to the inertial complex, though there an equivalent, but more complicated, gauge covariant version that we need not explicate.

Of course, spin appears in (139) because $\mathcal{T}^{\mu}$ is not a symmetric tensor. Indeed

$$
\left(\partial_{\nu} \underline{S}^{\nu}\right) \cdot \gamma^{\mu}=\partial_{\nu}\left(\underline{U}^{\sigma \nu} \wedge \gamma_{\sigma}\right) \cdot \gamma^{\mu}=\left(\underline{\mathcal{T}}^{\sigma} \wedge \gamma_{\sigma}\right) \cdot \gamma^{\mu}=\underline{\mathcal{T}}^{\mu}-\overline{\mathcal{T}}^{\mu}
$$

shows that spin divergence determines the skew symmetric part of $\underline{\mathcal{T}}^{\mu}$. When $\partial_{\nu} \underline{S}^{\nu}=0$, spin and orbital angular momentum are separately conserved. The existence of such a general spin conservation law has hitherto gone unnoticed. Of course, it is a "spin-off" of the superpotential. Indeed, the spin tensor is completely equivalent to the superpotential, and its interpretation as angular momentum may make it physically more perspicuous, as we see next.

To clarify the structure of the spin tensor, consider the expansion

$$
\begin{aligned}
\kappa \underline{S}^{\mu} & =\kappa \underline{U}^{\nu \mu} \wedge \gamma_{\nu}=\left[\left(\gamma^{\nu} \wedge \gamma^{\mu} \wedge \gamma^{\sigma}\right) \cdot \underline{\Omega}_{\sigma}\right] \wedge \gamma_{\nu} \\
& =\left[\gamma^{\nu}\left(\gamma^{\mu} \wedge \gamma^{\sigma}\right) \cdot \underline{\Omega}_{\sigma}-\gamma^{\mu}\left(\gamma^{\nu} \wedge \gamma^{\sigma}\right) \cdot \underline{\Omega}_{\sigma}+\gamma^{\sigma}\left(\gamma^{\nu} \wedge \gamma^{\mu}\right) \cdot \underline{\Omega}_{\sigma}\right] \wedge \gamma_{\nu} \\
& =-\gamma^{\mu} \wedge\left(\gamma^{\sigma} \cdot \underline{\Omega}_{\sigma}\right)+\gamma^{\sigma} \wedge\left(\gamma^{\mu} \cdot \underline{\Omega}_{\sigma}\right) \\
& =\gamma^{\sigma} \cdot\left(\gamma^{\mu} \wedge \underline{\Omega}_{\sigma}\right)-\gamma^{\mu} \cdot\left(\gamma^{\sigma} \wedge \underline{\Omega}_{\sigma}\right) \\
& =\underline{\Omega}^{\mu}+\left(\gamma^{\sigma} \cdot \underline{\Omega}_{\sigma}\right) \wedge \gamma^{\mu}-\gamma^{\mu} \cdot\left(\gamma^{\sigma} \wedge \underline{\Omega}_{\sigma}\right) .
\end{aligned}
$$

Therefore, the spin tensor is directly related to the superpotential and the connexion by

$$
\underline{S}^{\mu}=\underline{U}^{\nu \mu} \wedge \gamma_{\nu}=\kappa^{-1}\left(\gamma^{\sigma} \wedge \gamma^{\mu}\right) \times \underline{\Omega}_{\sigma}=\kappa^{-1} \underline{\Omega}^{\mu}+\frac{1}{2}\left[\underline{I} \wedge \gamma^{\mu}+(i \underline{H}) \cdot \gamma^{\mu}\right],
$$

where the "frame invariant" vectors are given by

$$
\underline{I}=-\gamma_{\mu} \cdot \underline{S}^{\mu} \quad \text { and } \quad i \underline{H}=-\gamma_{\mu} \wedge \underline{S}^{\mu} .
$$

This equivalence of spin to superpotential suggests that gravitational energymomentum is fundamentally rotational. Without taking a definitive stand on this issue, we can formally replace the superpotential by the spin tensor as the generator of the energymomentumn complex. Thus, using (142) in (135) we obtain

$$
\overline{\mathcal{T}}^{\mu}=\nabla \cdot\left[\underline{S}^{\mu}-\frac{1}{2}\left(\gamma_{\nu} \wedge \underline{S}^{\nu}\right) \cdot \gamma^{\mu}\right]
$$


We could solve (143) for $\underline{U}^{\nu \mu}$ as a function of $\underline{S}^{\mu}$, but that is unnecessary, as we have already replaced the superpotential by the spin tensor in the significant physical quantities.

Now let us turn to the formulation of integral conservation laws for inertial systems. Incorporation of integration theory with differential forms into geometric calculus has been expounded elsewhere [23]. Here we use only a fragment of the theory for non-null manifolds.

The differential on an $m$-dimensional submanifold of spacetime is a simple $m$-vector $d^{m} x$ tangent to the manifold at the point $x$, so it can be resolved into its magnitude $\left|d^{m} x\right|$ and its direction, represented by a unit $m$-vector field $I_{m}=I_{m}(x)$ on the manifold; thus,

$$
d^{m} x=I_{m}\left|d^{m} x\right| .
$$

For a coordinate system on the manifold, we can write

$$
\left|d^{m} x\right|=\left|\operatorname{detg}_{\mu \nu}\right|^{1 / 2} d x^{1} d x^{2} \ldots d x^{m},
$$

a familiar expression for the "volume element" in a "multiple integral."

Now let $M^{\mu}=M\left(\gamma^{\mu}\right)$ be any multivector-valued tensor of rank 1 on spacetime. For any $m$-dimensional submanifold of spacetime, the generalized Gauss' theorem (a version of Stokes' Theorem) can be expressed in the form

$$
\int \grave{M}(\grave{\partial})\left|d^{m} x\right|=\oint M\left(n^{-1}\right)\left|d^{m-1} x\right| .
$$

where

$$
\partial=I_{m}^{-1} I_{m} \cdot \nabla
$$

is the projection of $\nabla$ into the tangent space at each interior point, and at each boundary point $x$ the unit outward normal $n=n(x)$ is determined by

$$
I_{m}=I_{m-1} n .
$$

This theorem applies to manifolds of any signature. The effect of signature in the theorem is incorporated in the $n^{-1}$, which becomes $n^{-1}=n$ if $n^{2}=1$ or $n^{-1}=-n$ if $n^{2}=-1$.

The constant timelike vector $\gamma_{0}$ determines a 1-parameter family of spacetime hyperplanes $\mathcal{S}(t)$ satisfying the equation

$$
x \cdot \gamma_{0}=t ;
$$

In other words, $\mathcal{S}(t)$ is a surface of simultaneous $t$. Let $\mathcal{V}(t)$ be a convex 3dimensional region (submanifold) in $\mathcal{S}(t)$ which sweeps out a 4-dimensional region $\mathcal{M}$ in the time interval $t_{1} \leq t \leq t_{2}$. In this interval the 2-dimensional boundary $\partial \mathcal{V}(t)$ sweeps out a 3 -dimensional wall $\mathcal{W}$, so $\mathcal{M}$ is bounded by $\partial \mathcal{M}=\mathcal{V}\left(t_{1}\right)+\mathcal{V}\left(t_{2}\right)+\mathcal{W}$. 
Now, integrating the differential conservation law $\partial_{\mu} \mathcal{T}^{\mu}=\grave{\mathcal{T}}(\grave{\nabla})=0$ over the region $\mathcal{M}$, we obtain the integral conservation law

$$
\underline{P}\left(t_{2}\right)-\underline{P}\left(t_{1}\right)=\int_{t_{1}}^{t_{2}} d t \oint_{\partial \mathcal{V}(t)} \underline{\mathcal{T}}(n)\left|d^{2} x\right| .
$$

where the energymomentum vector $\underline{P}(t)=\underline{P}[\mathcal{V}(t)]$ for the region $\mathcal{V}(t)$ is defined by

$$
\underline{P}(t)=\int_{\mathcal{V}(t)} \underline{\mathcal{T}}\left(\gamma^{0}\right)\left|d^{3} x\right| .
$$

Introducing the superpotential with $\underline{\mathcal{T}}\left(\gamma^{0}\right)=\partial_{\nu} \underline{U}\left(\gamma^{0} \wedge \gamma^{\nu}\right)=\underline{\grave{U}}(\grave{\nabla})$, we can apply Gauss' theorem again to express the momentum as a boundary integral:

$$
\underline{P}(t)=\oint_{\partial \mathcal{V}(t)} \underline{U}(\mathbf{n})\left|d^{2} x\right|,
$$

where $\mathbf{n}=n \gamma_{0}=\gamma_{0} n^{-1}$ is the $3 \mathrm{D}$ outward normal. Note that the operator $\boldsymbol{\nabla}=\gamma^{0} \wedge \nabla$ is a projection of the $4 \mathrm{D}$ vector derivative onto the $3 \mathrm{D}$ manifold $\mathcal{V}(t)$, as required for applicability of Gauss' theorem to a submanifold.

The integral angular momentum conservation laws are derived in exactly the same way, simply by replacing the energymomentum tensor and its superpotential by the angular momentum tensor and its superpotential, so the steps need not be spelled out. The differential conservation laws for charge and other quantities can likewise be converted to integral conservation laws by analogous application of Gauss' theorem.

It should be noted that the total energymomentum vector $\underline{P}$ defined by (153) is in perfect accord with special relativity. In particular, a rigid displacement of the whole system contained in the region $\mathcal{M}$, as defined by the Poincaré transformation (36), induces the Lorentz rotation

$$
\underline{P} \quad \rightarrow \quad \underline{P}^{\prime}=L \underline{P} \tilde{L} .
$$

This, of course, is the most general transformation that leaves the flat space background invariant. Thus, the Poincare group is the kinematic group of the theory.

Recall from Section III that gauge theory gravity requires that the equations of physics be invariant under the Position Gauge Group (PGG) and covariant under the Local Rotation Gauge Group (LRGG). Factoring out the kinematic Poincaré group (PG), we can identify

$$
D G G \equiv P G G \otimes L R G G / P G .
$$

as the dynamical gauge group of the theory.

Finally, we note that the existence of position gauge invariant conservation laws is a consequence of the homogeneity and isotropy of the assumed flat spacetime background. Rosen [16] has emphasized that spaces of constant curvature 
are also homogeneous and isotropic, so our analysis of conservation laws readily generalizes to those cases. This eliminates the objection to GTG that it is does not allow a closed topology for the universe. That is a subject to be addressed at another time.

\section{Assessing the Energymomentum Complex}

The deciding factor in any formulation of the energymomentum complex is physical significance. To assess the inertial energymomentum complex of the preceding section, we examine its implications for a sample of significant gauge tensor solutions to Einstein's equation. For any given gauge tensor, the connexion $\Omega_{\mu}$ can be calculated from (61) with (62) and (63), which for an inertial frame can be put in the form

$$
2 \Omega_{\mu}=\bar{h}\left[\gamma^{\nu} \wedge \gamma^{\sigma} g_{\nu} \cdot \partial_{\mu} g_{\sigma}-\nabla \wedge\left(g_{\mu \nu} \gamma^{\nu}\right)\right]
$$

where $g^{\nu} \wedge g^{\alpha}=\bar{h}\left(\gamma^{\nu} \wedge \gamma^{\alpha}\right)$ has been used. Therefrom, the superpotential $U^{\mu \nu}$ and all other features of the inertial energymomentum complex can be obtained by direct calculation.

A good test bed is the class of Kerr-Schild solutions, which Doran and Lasenby [22] have thoroughly studied with STA techniques. This class includes the important spherically-symmetric Schwarzschild, Reissner-Nordstrom and Viadya solutions as well as the axially-symmetric Kerr solution. Doran and Lasenby show that the Einstein tensor itself is a suitable energymomentum tensor for this class of solutions. That might be just a lucky accident, because their choice happens to be identical to our transposed energymomentum tensor (135) for this class. We can conclude immediately that their analysis of Kerr-Schild field properties is fully consistent with our characterization of the inertial energymomentum complex, though it does not validate the complex conclusively. Nevertheless, it is instructive to reconsider the Kerr-Schild energymomentum complex from the ground up. In the following analysis, we adhere closely to the notation in [22], which can be consulted for more details.

For an inertial frame, a Kerr-Schild gauge tensor has the general form

$$
g_{\nu}=\underline{h}^{-1}\left(\gamma_{\nu}\right)=\gamma_{\nu}+l_{\nu} l, \quad \text { with } \quad l^{2}=0
$$

and $l_{\nu}=l \cdot \gamma_{\nu}$. Consequently, the metric tensor has the form $g_{\sigma \nu}=\eta_{\sigma \nu}-2 l_{\sigma} l_{\nu}$ and we easily calculate that

$$
g_{\nu} \cdot \partial_{\mu} g_{\sigma}=g_{\sigma} \cdot \partial_{\mu} g_{\nu}
$$

[Recall from the end of Section V that condition (159) implies equivalence of Møller and Einstein energymomentum complexes. Therefore, our analysis of Kerr-Schild fields cannot provide grounds to discriminate between them, though we have seen that Møller's complex is much to be preferred on theoretical grounds.] 
Continuing our analysis, we find from (158) that

$$
g^{\nu}=\bar{h}\left(\gamma^{\nu}\right)=\gamma^{\nu}-l^{\nu} l,
$$

whence, for any $k$-vector $K$,

$$
\bar{h}(K)=K-(K \cdot l) l .
$$

In particular, for the unit pseudoscalar we have $(i \cdot l) l=(i l) l=0$, so

$$
\bar{h}(i)=i,
$$

and from (42) we conclude that $g=h=\operatorname{det}(\bar{h})=1$. Inserting these details into (157), we obtain a specific form for the Kerr-Schild connexion:

$$
\Omega_{\mu}=\bar{h}\left[\nabla \wedge\left(l_{\mu} l\right)\right]=\nabla \wedge\left(l_{\mu} l\right)+l_{\mu} l \wedge(l \cdot \nabla l) .
$$

Following [22], with little loss of generality we restrict our considerations to the case $l \wedge(l \cdot \nabla l)=0$ even inside matter, so the connexion (163) reduces to the simple form

$$
\Omega_{\mu}=\nabla \wedge\left(l_{\mu} l\right)
$$

Moreover, by the same argument $\Omega_{\mu}=g \underline{h}\left(\Omega_{\mu}\right)=\Omega_{\mu}$, so we can dispense with the underbars in the previous section. Equation (164) has the obvious property

$$
\frac{1}{2} \kappa i H \equiv \gamma^{\mu} \wedge \Omega_{\mu}=\nabla \wedge(l \wedge l)=0 .
$$

Hence, from (142) the spin tensor is given by

$$
\kappa S^{\mu}=\gamma^{\sigma} \cdot\left(\Omega_{\sigma} \wedge \gamma^{\mu}\right)=\grave{l} \cdot\left(\grave{\nabla} \wedge \grave{l} \wedge \gamma^{\mu}\right)=\partial_{\nu}\left[l \cdot\left(l \wedge \gamma^{\mu} \wedge \gamma^{\nu}\right)\right],
$$

where the accent indicates differentiation by $\grave{\nabla}$ to the left as well as to the right. From (145) the energymomentum tensor is therefore given by

$$
\overline{\mathcal{T}}^{\mu}=\nabla \cdot S^{\mu}=\nabla \cdot\left[\grave{l} \cdot\left(\grave{\nabla} \wedge \grave{l} \wedge \gamma^{\mu}\right)\right]=\partial_{\nu} \partial_{\alpha}\left[\left(\gamma^{\alpha} \wedge l\right) \cdot\left(l \wedge \gamma^{\mu} \wedge \gamma^{\nu}\right)\right] .
$$

To incorporate further details, we need to evaluate the connexion for the specific special cases.

To describe spherically-symmetric solutions in an inertial system specified by the timelike vector $\gamma_{0}$, we introduce the "spacetime split"

$$
x \gamma_{0}=x \cdot \gamma_{0}+x \wedge \gamma_{0}=t+r \boldsymbol{\sigma}_{r} .
$$

where $t=x \cdot \gamma_{0}=x \cdot \gamma^{0}$ is coordinate time,

$$
r=\left|x \wedge \gamma_{0}\right|
$$


is the radial coordinate to the spacetime point $x$, and the radial direction is described by

$$
\boldsymbol{\sigma}_{r}=e_{r} \gamma_{0} \quad \text { with } \quad e_{r}=\partial_{r} x=-e^{r}=-\nabla r .
$$

For spherically-symmetric solutions the null vector $l$ can be rescaled to

$$
l=e \sqrt{\varphi} \quad \text { with } \quad e=\gamma_{0}-e_{r}=\nabla(t-r)
$$

and $\varphi=\varphi(r)$. Inserting this into (164) and replacing $\gamma_{\mu}$ with a constant vector $a$, we get the connexion in the more explicit form

$$
\Omega(a)=\nabla \wedge(e \varphi e \cdot a)=\nabla(\varphi e \cdot a) \wedge e,
$$

where the last equality is a consequence of

$$
\nabla \wedge e=-\nabla \wedge e_{r}=\nabla \wedge \nabla r=0
$$

Before continuing, it is worth reviewing some techniques for evaluating vector derivatives.

From the definitions (34) and (35), we evaluate basic derivatives

$$
\nabla x \cdot a=a \cdot \nabla x=a \quad \text { and } \quad \nabla x=4 .
$$

Therefrom, other derivatives can be calculated. Thus

$$
\nabla(x \wedge a)=\nabla(x a-x \cdot a)=3 a .
$$

From $e_{r}=\left(x \wedge \gamma_{0}\right) \gamma_{0} / r$ we calculate

$$
\nabla e=-\nabla e_{r}=-\frac{2}{r}=\nabla \cdot e
$$

and

$$
\nabla e \cdot a=-\nabla a \cdot e_{r}=\frac{1}{r}\left[\left(\gamma_{0} \wedge a\right) \gamma_{0}-a \cdot e_{r} e_{r}\right]=\frac{1}{r}\left(a \cdot e e_{r}-a+a \cdot \gamma_{0} e\right) .
$$

We will also need

$$
\nabla\left(\frac{\sigma_{r}}{r^{2}}\right)=\nabla \cdot\left(\frac{x \wedge \gamma_{0}}{r^{3}}\right)=4 \pi \delta^{3}\left(x \wedge \gamma_{0}\right) \gamma_{0}=\gamma_{0} \nabla\left(\frac{e_{r}}{r^{2}}\right),
$$

where $\delta^{3}\left(x \wedge \gamma_{0}\right)=\delta^{3}(\mathbf{x})$ is the usual $3 \mathrm{D}$ delta function for the position variable $\mathbf{x}=x \wedge \gamma_{0}$.

Returning now to the connexion (172), we use (177) to complete the differentiation and obtain the general form

$$
\Omega(a)=\left[\left(\frac{\varphi}{r}-\partial_{r} \varphi\right) a \cdot e e_{r}-\frac{\varphi a}{r}\right] \wedge e .
$$


For the Schwarzschild solution $\varphi=M / r$, so the connexion has the specific form

$$
\Omega(a)=\frac{M}{r^{2}}\left[2 a \cdot e e_{r}-a\right] \wedge e,
$$

or, equivalently,

$$
\Omega_{\mu}=\frac{M}{r^{2}}\left[2 \gamma_{\mu} \cdot e \boldsymbol{\sigma}_{r}+e \wedge \gamma_{\mu}\right] .
$$

Whence we obtain the inertia vector

$$
I=2 \kappa^{-1} \gamma^{\mu} \cdot \Omega_{\mu}=-\frac{2 M}{\kappa r^{2}} e .
$$

Its divergence is

$$
\nabla \cdot I=\frac{2 M}{\kappa} \nabla \cdot\left(\frac{e_{r}}{r^{2}}\right)=M \delta^{3}\left(x \wedge \gamma_{0}\right) .
$$

This connection with mass is the motivation for the name given to the vector field I.

Using (127), from (181) we obtain an explicit form for the superpotential:

$$
U^{\mu \nu}=\frac{2 M}{\kappa r^{2}}\left(\gamma^{\mu} \wedge \gamma^{\nu}\right) \cdot \sigma_{r} e,
$$

and therefrom the energymomentum tensor:

$$
\mathcal{T}^{\mu}=\partial_{\nu} U^{\mu \nu}=\frac{2 M}{\kappa} \gamma^{\mu} \cdot\left[\nabla \cdot\left(\frac{\sigma_{r}}{r^{2}}\right)\right] e=\gamma^{\mu} \cdot \gamma_{0} M \delta^{3}\left(x \wedge \gamma_{0}\right) e .
$$

Its adjoint is

$$
\overline{\mathcal{T}}^{\mu}=\nabla \cdot\left[\frac{2 M}{\kappa r^{2}} \boldsymbol{\sigma}_{r} \gamma^{\mu} \cdot e\right]=\frac{2 M}{\kappa} \gamma^{\mu} \cdot e \nabla \cdot\left(\frac{\boldsymbol{\sigma}_{r}}{r^{2}}\right) .
$$

This is identical to the expression found by Doran and Lasenby [22]. The spin tensor has the form

$$
S^{\mu}=\gamma_{\nu} \wedge U^{\mu \nu}=\frac{2 M}{\kappa r^{2}}\left(\gamma^{\mu} \cdot \sigma_{r}\right) \wedge e,
$$

and its divergence is

$$
\partial_{\mu} S^{\mu}=\gamma_{\nu} \wedge U^{\mu \nu}=\frac{2 M}{\kappa}\left[\nabla \cdot\left(\frac{\sigma_{r}}{r^{2}}\right)\right] \wedge e=M \delta^{3}\left(x \wedge \gamma_{0}\right) \gamma_{0} \wedge e,
$$

which is equal to $\gamma_{\mu} \wedge \mathcal{T}^{\mu}$ as required. Note that it vanishes outside the origin, in keeping with symmetry of the gravitational energymomentum tensor.

The energymomentum integral (153) is easily evaluated from (184). For a sphere with outward normal $\mathbf{n}=\boldsymbol{\sigma}_{r}$ we obtain

$$
P=\oint_{\partial \mathcal{V}} U(\mathbf{n})\left|d^{2} x\right|=\oint \frac{2 M}{\kappa r^{2}}(1-\mathbf{n}) \gamma_{0} r^{2} d^{2} \Omega=M \gamma_{0},
$$


as required for a point particle with mass $M$ at rest.

The Riessner-Nordstrom solution for the gravitational field of a point charge at rest differs from the Schwarzschild solution only in the "scalar potential," which has the form

$$
\varphi=\frac{M}{r}-\frac{q^{2}}{\kappa r^{2}} \equiv \varphi_{1}+\varphi_{2} .
$$

Consequently, the the connexion (179) is expressible as a superposition

$$
\Omega(a)=\Omega_{1}(a)+\Omega_{2}(a),
$$

where $\Omega_{1}(a)$ is the Schwarzschild connection, which we have already considered. With $\partial_{r} \varphi_{2}=-2 \varphi_{2} / r$ in (179), we find that the contribution of the electric field is given by the connection

$$
\Omega_{2}(a)=\frac{\varphi_{2}}{r}\left(3 a \cdot e e_{r}-a\right) \wedge e=\frac{-q^{2}}{\kappa r^{2}}\left(3 a \cdot e \boldsymbol{\sigma}_{r}+e \wedge a\right) .
$$

Whence we find that the inertia vector vanishes:

$$
I_{2}=2 \kappa^{-1} \gamma^{\mu} \cdot \Omega_{2}\left(\gamma_{\mu}\right)=\frac{\varphi_{2}}{\kappa r}\left[\left(3 e \cdot \sigma_{r}+\gamma^{\mu} \cdot\left(e \wedge \gamma_{\mu}\right)\right]=0,\right.
$$

and the superpotential has the form

$$
U_{2}^{\mu \nu}=\kappa^{-1}\left(\gamma^{\mu} \wedge \gamma^{\nu}\right) \cdot \Omega_{2}\left(\gamma_{\lambda}\right) \gamma^{\lambda}=\frac{\varphi_{2}}{\kappa r}\left[3\left(\gamma^{\mu} \wedge \gamma^{\nu}\right) \cdot \sigma_{r} e+\left(\gamma^{\mu} \wedge \gamma^{\nu}\right) \cdot e\right]
$$

However, it is more convenient to use (135) to get

$$
\begin{aligned}
\kappa \overline{\mathcal{T}}_{2}(a)=\nabla \cdot \Omega_{2}(a) & =\frac{q^{2}}{\kappa} \nabla \cdot\left[e \wedge \nabla\left(\frac{e \cdot a}{r^{2}}\right)\right] \\
& =\frac{q^{2}}{\kappa} \nabla \cdot\left[\frac{e \wedge a-3 e \cdot a \boldsymbol{\sigma}_{r}}{r^{3}}\right] .
\end{aligned}
$$

This result agrees with [22], which gives its components such as

$$
\kappa \overline{\mathcal{T}}_{2}^{0}=\frac{q^{2}}{\kappa} \nabla \cdot\left(\frac{-2 \boldsymbol{\sigma}_{r}}{r^{3}}\right)=\frac{q^{2}}{4 \pi r^{2}} \gamma_{0},
$$

and shows that it is equivalent to the standard form for the electromagnetic energymomentum tensor:

$$
\overline{\mathcal{T}}_{2}^{\mu}=-\frac{1}{2} \mathcal{F} \gamma^{\mu} \mathcal{F} \quad \text { where } \quad \mathcal{F}=\frac{q \boldsymbol{\sigma}_{r}}{4 \pi r^{2}}
$$

is the Coulomb field of the point charge.

Following [22], we can investigate the singularity at the origin by considering the integral of the total energymomentum tensor $\overline{\mathcal{T}}(a)=\overline{\mathcal{T}}_{1}(a)+\overline{\mathcal{T}}_{2}(a)$ over a 
sphere centered at the origin; thus

$$
\begin{aligned}
\int\left|d^{3} x\right| \overline{\mathcal{T}}(a) & =\int\left|d^{3} x\right| \nabla \cdot\left[\frac{2 M}{\kappa r^{2}} e \cdot a \boldsymbol{\sigma}_{r}+\frac{q^{2}}{\kappa^{2} r^{3}}\left(e \wedge a-3 e \cdot a \boldsymbol{\sigma}_{r}\right)\right] \\
& =-\oint d^{2} \Omega e_{r} \cdot\left[\frac{2 M}{\kappa} e \cdot a \boldsymbol{\sigma}_{r}+\frac{q^{2}}{\kappa^{2} r}\left(e \wedge a-3 e \cdot a \boldsymbol{\sigma}_{r}\right)\right] \\
& =\left(M-\frac{q^{2}}{\kappa r}\right) a \cdot \gamma_{0} \gamma_{0}+\frac{q^{2}}{3 \kappa r}\left(a \wedge \gamma_{0}\right) \gamma_{0} .
\end{aligned}
$$

Thus the total energymomentum in the sphere is

$$
P=\int\left|d^{3} x\right| \overline{\mathcal{T}}^{0}=\left(M-\frac{q^{2}}{\kappa r}\right) \gamma_{0} .
$$

As observed in [22], this tells us that the electromagnetic contribution to the energy is negative, and it vanishes when the integral is extended to infinity. Therefore, inclusion of the gravitational field has removed the divergent selfenergy of the classical electromagnetic field!

The gauge tensor for Vaidya's "shining star" solution has the form [22]

$$
\bar{h}(a)=a+\frac{\mu(t-r)}{r} a \cdot e_{+} e_{+} \quad \text { where } \quad e_{+}=\gamma_{0}+e_{r} .
$$

This is nearly the same as the the Schwarzschild gauge tensor, so it leads to a connexion of the same form as (172):

$$
\Omega(a)=\nabla \wedge\left[\frac{\mu}{r} e_{+} \cdot a e_{+}\right]=\mu\left[\nabla\left(\frac{a \cdot e_{+}}{r}\right)\right] \wedge e_{+}
$$

where $\nabla \mu=e_{+} \partial_{t} \mu$ was used to kill the derivative of $\mu$ in the last step. Consequently, it differentiates to a perfect analog of the Schwarzschild form (181):

$$
\Omega_{2}\left(\gamma_{\mu}\right)=\frac{\mu}{r^{2}}\left[2 \gamma_{\mu} \cdot e_{+} \boldsymbol{\sigma}_{r}+e_{+} \wedge \gamma_{\mu}\right]
$$

We can skip other details that are similar to the Schwarzschild case. However, one significant difference is that the energymomentum tensor no longer vanishes outside the source. Indeed, in analogy to (186) the adjoint tensor has the form

$$
\overline{\mathcal{T}}^{\mu}=\nabla \cdot\left[\frac{2 \mu}{\kappa r^{2}} \boldsymbol{\sigma}_{r} \gamma^{\mu} \cdot e_{+}\right]=-\frac{2 \partial_{t} \mu}{\kappa r^{2}} \gamma^{\mu} \cdot e_{+} e_{+}
$$

This is identical to the result in [22], which notes that it describes a steady loss of mass from the source of the gravitational field.

For the Kerr solution we adopt the notation of [22] to write the connexion (164) in the form

$$
\Omega(a)=M \nabla \wedge[\alpha(a \cdot n) n]=M[\alpha(\nabla a \cdot n) \wedge n+a \cdot n \nabla \wedge(\alpha n)],
$$

where $n=(1-\mathbf{n}) \gamma_{0}$ is a null vector so $\mathbf{n}=\gamma_{0} \wedge n$ is a unit bivector, and we anticipate that the source has mass $M$. The functional forms of $\alpha=\alpha(\mathbf{x})$ 
and $\mathbf{n}=\mathbf{n}(\mathbf{x})$ are determined by Einstein's equation, which [22] shows can be reduced (outside the source singularity) to the equations

$$
\begin{gathered}
\boldsymbol{\nabla} \mathbf{n}=\boldsymbol{\nabla} \cdot \mathbf{n}+\boldsymbol{\nabla} \wedge \mathbf{n}=2(\alpha-i \beta \mathbf{n}) \\
\boldsymbol{\nabla}^{2} \gamma=0 \quad \text { with } \quad\left(\boldsymbol{\nabla} \gamma^{-1}\right)^{2}=1
\end{gathered}
$$

with scalar function $\beta=\beta(\mathbf{x})$ incorporated into a formally "complex" funcction $\gamma \equiv \alpha+i \beta$, and $\boldsymbol{\nabla}=\gamma_{0} \wedge \nabla=\partial_{\mathbf{x}}$.

The axially-symmetric solution of Laplace's equation (190) with the right boundary conditions is

$$
\gamma=\left[\rho^{2}+(z-i L)^{2}\right]^{-\frac{1}{2}}
$$

where $z \equiv x \cdot \gamma^{3}=\mathbf{x} \cdot\left(\gamma_{3} \gamma_{0}\right)$ and $\rho^{2}=\mathbf{x}^{2}-z^{2}$ with scalar constant $L$ as angular momentum of the source. This solution has a disk singularity in the $z=0$ plane that is thoroughly studied in [22].

For our computations we need the following definitions and derived relations:

$$
\begin{gathered}
\boldsymbol{\sigma}_{\gamma} \equiv \nabla \gamma^{-1}=\gamma\left(\mathbf{x}-L i \gamma_{3} \gamma_{0}\right) \\
\nabla \wedge(\alpha n)=-\nabla \gamma=\gamma^{2} \boldsymbol{\sigma}_{\gamma}, \\
n \boldsymbol{\sigma}_{\gamma}=n=-\boldsymbol{\sigma}_{\gamma} n, \\
n \wedge \nabla a \cdot n=n\left(a \cdot n \gamma_{0}-a\right) \gamma=\left[n \wedge \gamma_{0} a \cdot n+a \wedge n\right] \gamma .
\end{gathered}
$$

Using these results, we can express the Kerr connexion (204) in the explicit form

$$
\Omega(a)=M \gamma\left[a \cdot n\left(\gamma \boldsymbol{\sigma}_{\gamma}+\alpha \gamma_{0} \wedge n\right)+\alpha n \wedge a\right]
$$

As a check, it may be noted that for $L=0$ this reduces to the Schwarzschild connexion (180) with $\gamma=\alpha=r^{-1}$ and $\boldsymbol{\sigma}_{\gamma}=\boldsymbol{\sigma}_{r}=\mathbf{n}$.

The spin tensor is given by

$$
\kappa S(a)=\gamma^{\sigma} \cdot\left(\Omega_{\sigma} \wedge a\right)=M\left[a \cdot n\left(\gamma^{2} \boldsymbol{\sigma}_{\gamma}+(\alpha \gamma) \gamma_{0} \wedge n\right)+(i \beta \gamma) n \wedge a\right]
$$

We are especially interested in the spin flux in the $\gamma_{0}$ direction:

$$
\kappa S^{0}=M\left[\gamma^{2} \boldsymbol{\sigma}_{\gamma}+\left(\gamma \gamma^{*}\right) \gamma_{0} \wedge n\right]=M\left[-\nabla \gamma+\left(\alpha^{2}+\beta^{2}\right) \mathbf{n}\right] .
$$

Whence the energymomentum flux is

$$
\overline{\mathcal{T}}^{0}=\nabla \cdot S^{0}=\kappa^{-1} M \nabla \cdot\left[-\nabla \gamma+\left(\alpha^{2}+\beta^{2}\right) \mathbf{n}\right]
$$


As defined in (153), the energymomentum vector for a spacelike region $\mathcal{V}=\mathcal{V}(t)$ is given by

$$
\begin{aligned}
P & =\int_{\mathcal{V}}\left|d^{3} x\right| \overline{\mathcal{T}}^{0}=\oint_{\partial \mathcal{V}}\left|d^{2} x\right| \hat{e}_{u} \cdot S^{0} \\
& =\kappa^{-1} M \oint\left|d^{2} x\right| \hat{e}_{u} \cdot\left[-\nabla \gamma+\left(\alpha^{2}+\beta^{2}\right) \mathbf{n}\right]=M \gamma_{0},
\end{aligned}
$$

the expected result. Though the integral is independent of the the region $\mathcal{V}$ as long as it contains the singularity, it is most easily evaluated for an oblate spheroid with outward unit normal $\hat{e}_{u}$, which reflects the symmetry of the source. Details of the integration are given in [22].

The Inertia vector is given by

$$
I=S^{\mu} \cdot \gamma_{\mu}=2 \kappa^{-1} \partial_{\mu}\left(l^{\mu} l\right)=-2 \kappa^{-1} M\left(\alpha^{2}+\beta^{2}\right) n .
$$

Whence, by a similar integration, we obtain

$$
\int_{\mathcal{V}}\left|d^{3} x\right| \operatorname{Tr} \mathcal{T}=\int\left|d^{3} x\right| \nabla \cdot I=-2 \kappa^{-1} M \oint_{\mathcal{V}}\left|d^{2} x\right|\left(\alpha^{2}+\beta^{2}\right) \hat{e}_{u} \cdot n=M,
$$

the same as the result for the Schwarzschild case.

Since the energymomentum tensor is symmetric, the orbital and spin angular momenta are separately conserved. They are nevertheless related by

$$
\overline{\mathcal{T}}(a) \wedge x=[\nabla \cdot S(a)] \wedge x=\partial_{\mu}\left[\left(\gamma^{\mu} \cdot S(a)\right) \wedge x\right]+2 S(a) .
$$

Accordingly, the orbital angular momentum for the region $\mathcal{V}$ is given by

$$
\int_{\mathcal{V}}\left|d^{3} x\right| \overline{\mathcal{T}}^{0} \wedge x=2 \int_{\mathcal{V}}\left|d^{3} x\right| S^{0}=M \operatorname{Li} \gamma_{3} \gamma_{0}
$$

Again (except, perhaps, for the factor 2) this is exactly what we expect, and details of the integration are given in [22] (though the present formulation introduces some simplifications).

The present study of the energymomentum complex for Kerr geometry is completed by the thorough analysis of the Kerr ring singularity given in [22].

\section{Conclusions}

It took almost a quarter century to identify the superpotential in Einstein's energymomentum complex [21], and another quarter century to scope out the range of alternative superpotentials until Møller realized the unique advantages of a "tetrad superpotential" [12]. And to this day, Møller's contribution is generally overlooked. One reason for the long time lag may be obscurity and complexity of the tensor and tetrad formulations. We saw in Section V that, with a new unitary formulation of Einstein's tensor, STA greatly simplifies and clarifies the 
formulation and analysis of superpotentials. Then we saw that the canonical split of Einstein's tensor in Section VI brings even greater simplifications and surprising new insights into the structure of the "energymomentum complex."

We have seen that the energymomentum superpotential (??) is manifestly gauge invariant, and it generates an energymomentum conservation law that is consistent with the requirements of special relativity. Nevertheless, more work is needed before we can be sure that this is a satisfactory solution to the longstanding problem of energymomentum conservation in general relativity. Though our application of the "inertial complex" to Kerr-Schild fields provides significant confirmation, it is not even sufficient to distinguish the canonical complex from Einstein's original energymomentum complex. Further tests on solutions on Einstein's equation are needed, especially for gravitational radiation and the new ideas about spin. The effect of local gauge transformations, especially on physical interpretion, remains to be studied. The gauge can be fixed in many ways, such as boundary conditions at sources or globally. It remains to be seen if some gauges have special physical significance.

Finally, it should be observed that the formulation of the canonical energymomentum complex in Section V is sufficiently general to include torsion, so

it is immediately applicable to Dirac theory [3], where it raises new questions about the relation of mass and spin in sources to the gravitational field.

\section{References}

[1] D. Hestenes, "Gauge Theory Gravity with Geometric Calculus," Am. J. Phys. ??: ??? (2005).

[2] C. Doran \& A. Lasenby, Geometric Algebra for Physicists (Cambridge U Press, Cambridge, 2003).

[3] A. Lasenby, C. Doran, \& S. Gull, "Gravity, gauge theories and geometric algebra," Phil. Trans. R. Lond. A 356: 487-582 (1998).

[4] D. Hestenes, Space-Time Algebra, (Gordon \& Breach, New York, 1966).

[5] D. Hestenes \& G. Sobczyk, CLIFFORD ALGEBRA to GEOMETRIC CALCULUS, a Unified Language for Mathematics and Physics (Kluwer Academic, Dordrecht/Boston, 1986).

[6] H. Weyl, "Elektron and Gravitation I." Zeitshrift für Physik, 56: 330 (1929).

[7] A. Einstein, "Hamilton's Principle and the General Theory of Relativity," Sitzungsber.D. Preuss. Akad. D. Wiss., 1916. English translation in The Principle of Relativity (Dover, 1923).

[8] J. Goldberg, "Invariant Transformations, Conservation Laws, and EnergyMomentum." In A. Held, General Relativity and Gravitation, Vol. I (Plenum, New York, 1980). pp. 469-489. 
[9] J. Winnicour, "Angular Momentum in General Relativity." In A. Held, General Relativity and Gravitation, Vol. II (Plenum, New York, 1980). pp. $71-95$.

[10] The early history of conservation laws in GR is surveyed by C. Cattani \& M. DeMaria, "Conservation Laws and Gravitational Waves in General Relativity," In J. Earman, M. Janssen \& J. Norton (Eds.) The Attraction of Gravitation: New Studies in the History of General Relativity. Birkhäuser, Boston, 1993).

[11] L. Landau \& E. Lifshitz, The Classical Theory of Fields, (Addison-Wesley, Reading, MA 1971.

[12] C. Møller, "Momentum and Energy in General Relativity and Gravitational Radiation," Mat. Fys. Medd. Dan. Vid. Selsk. 34 (no. 3), 1-67 (1964). This completes a long analysis in several articles beginning in 1958. The analysis is summarized in: C. Moller, "Survey of Investigations on the Energymomentum Complex in General Relativity," Mat. Fys. Medd. Dan. Vid. Selsk. 35 (no. 3), 1-14 (1966); also in C. Moller, "The Four-Momentum of an Insular System in General Relativity," Nuclear Physics 57: 330-338 (1964).

[13] S. Gupta, "Einstein's and Other Theories of Gravitation," Rev. Mod. Phys. 29: 334-336 (1957).

[14] W. Thirring, "An Alternative Approach to the Theory of Gravitation," Annals of Physics 16: 96-117 (1961).

[15] R. Feynman, Feynman Lectures on Gravitation (Addison-Wesley, Reading, 1995).

[16] N. Rosen, "A Theory of Gravitation," Annals of Physics 84 455-473 (1974). This is representative of many papers by Rosen.

[17] M. Israelit, "A Gauge-Covariant Bimetric Tetrad Theory of Gravitation and Electromagnetism," Found. Physics 19 33-55 (1989).

[18] S. Babak \& L. Grishchuk, "Energy-momentum tensor for the gravational field," Phys. Rev. D 61, 1-18 (1999).

[19] D. Hestenes, "Spacetime physics with geometric algebra," Am. J. Phys. 71 691-704 (2003).

[20] Much more literature on extensions and applications of Geometric Algebra and Calculus can be accessed from the websites $<$ http://modelingnts.la.asu.edu $>$ and $<$ http://www.mrao.cam.ac.uk/ clifford/>.

[21] Ph. Freud, "On the Expression of the Total Energy and the Total Momentum of a Material System in the Theory of General Relativity," Ann. Math. Princeton 40 417-419 (1939). 
[22] C. Doran \& A. Lasenby, "Integral Equations, Kerr-Schild Fields and Gravitational sources." (arXiv:gr-qc/0404081 v1 19 Apr 2004).

[23] D. Hestenes, "Differential Forms in Geometric Calculus." In F. Brackx, R. Delanghe, and H. Serras (eds), Clifford Algebras and their Applications in Mathematical Physics (Kluwer Academic, Dordrecht/Boston, 1993). pp. 269-285.

[24] C. Misner, K. Thorne \& J. Wheeler, Gravitation (Freeman, San Francisco, 1973). 